(C) Masson, Paris, 1980.

\title{
Oncomiracidiums et phylogenèse des Monogenea (Plathelminthes)
}

\author{
$1^{\text {re }}$ Partie : Développement post-larvaire
}

\begin{abstract}
A. LAMBERT
Laboratoire de Parasitologie comparée, Université des Sciences et Techniques du Languedoc, place E.-Bataillon, F 34060 Montpellier Cedex
\end{abstract}

RESUME. Cet article complète et synthétise les résultats des travaux publiés énumérés pages 168 à 170 .

Il comprend quatre chapitres dont les deux premiers sont envisagés ici ; il s'agit de :

- Matériel et méthodes :

Les techniques utilisées sont précisées, en particulier les imprégnations argentiques des oncomiracidiums des Monogènes marins.

- Développement post-larvaire et morphogénèse du hapteur des Dactylogyridea (Monogenea, Monopisthocotylea) :

Nous développons et discutons successivement les problèmes suivants :

1. Modalités d'infestation et installation du parasite chez le Poisson-hôte.

2. Morphogénèse du hapteur où sont étudiés l'origine des hamuli et les transformations adaptatives de cet organe de fixation. (fin de la $1^{\text {re }}$ partie).

La deuxième partie, à paraître, sera constituée par les deux chapitres suivants :

- les structures argyrophiles des oncomiracidiums.

- L'évolution des Monogenea.

\section{Oncomiracidia and phylogenesis of Monogenea (Plathelminthes).}

Part one: Post-larval development.

SUMMARY. In order to present this work, we insist on the necessity, for a better understanding of the evolution of the Monogeneans, of studying another system than the haptor. In fact, it is this organ which, in adults, then in larvae, was the basis of

Accepté le 19 novembre 1979. 
research on systematics and phylogenesis of these Plathelminthes. Because of the attachment systems affording strong adaptative variations, it was important, according to us, to complete the researches by a study of structures which were unlikely to be modified by parasitism and less sensitive than haptor to adaptative phenomenons.

For these reasons, we directed our work toward ciliated cells and oncomiracidium sensillae.

This article, which completes and synthesizes the results published in works enumerated on pages 168 to 170 , is divided into four chapters that we summarize below.

\section{Experimental methods.}

The techniques of study used in laboratory and in the field are examined. The technique of silver impregnation established for oncomiracidiums of marine Monogeneans is explained and discussed.

\section{Post larval development and morphogenesis of haptor in Dactylogyridea (Mono- genea, Monopisthocotylea).}

We expose the following problems successively:

1. Modes of infestation of the host-fish by oncomiracidia.

We bring additional evidences of a initial cutaneous contact of the host by oncomiracidia followed by a migration to the adult habitat. The relative importance of cutaneous and buccal infestation, through the respiratory pathway, is discussed.

2. The morphogenesis of haptor in post larva.

After having shown, from precise examples, the body origin of hamuli in several Ancyrocephalidae, we propose the hypothesis of a "generalized migration of oncoblastes in Dactylogyridea". This allow us to show the common process of the migration in Calceostomatidae, Ancyrocephalidae, Dactylogyridae and Tetraonchidae.

They are variations due to adaptative transformations of the haptor relative to the process of attachment and to the habitat. They essentially consist in species attached between two gill lamellae, in a passage, on the dorsal side, of two pairs of larval hooklets. We have shown that it was not the rotation of dorsal hamuli that causes this change for they are, from their body origin, dorsally directed.

In species which the attachment is ventral and which possess dorsal hamuli, the direction of larval hooklet is not modified by hamuli.

(End of part one).

- Argyrophil structures of oncomiracidia and their post-larval evolution.

- Evolution of Monogenea.

These 2 chapters which form the last part of the article are to be published later. 


\section{Introduction}

Les Monogènes sont des Plathelminthes essentiellement ectoparasites de Poissons. Ils se caractérisent par un organe de fixation particulier, différencié dans la région postérieure du corps: le hapteur. Cet organe est la pièce maîtresse dont dépend l'existence même du parasite car il permet la réalisation du couple hôte-parasite, véritable entité biologique.

Au cours de l'évolution, cet organe de fixation s'est adapté à des microhabitats variés: la peau, les écailles placoïdes, les branchies, les branchiospines, et parfois même la cavité générale et l'estomac... Pour un microhabitat donné, les branchies par exemple, où ces parasites sont très fréquents, il existe une variété extrême de systèmes d'accrochage (crochets dont le nombre et la morphologie varient beaucoup, pinces, ventouses, pseudohapteurs...) ; chacun représente un mode de fixation précis.

Du fait de sa diversité comme organe d'attachement, le hapteur a suscité de nombreuses recherches car son étude débouchait sur les grands problèmes biologiques de Parasitologie fondamentale, comme ceux relatifs à l'adaptation à l'ectoparasitisme, à la spécificité parasitaire, aux relations hôtes-parasites, etc...

On peut distinguer deux grandes périodes dans l'histoire de la recherche sur les Monogènes :

- Une première époque, des origines aux années 1955-1957, a vu un effort essentiellement axé sur l'étude des adultes, lorsque le parasite est déjà installé sur son hôte. Conjointement aux recherches classiques sur la morpho-anatomie générale, on a porté une attention particulière au hapteur. Ainsi, les premières classifications sont surtout basées sur l'étude de cet organe (Price, 1937-1962 ; Sproston, 1946 : Hargis, 19551957). Il en est ressorti la démonstration de la grande diversité des systèmes d'attachement dont nous venons de parler.

- A partir des années 1955-1957, les chercheurs (Euzet, 1955 et Bychowsky, 1957) ont étudié le stade larvaire libre, nommé oncomiracidium par Llewellyn (1957 a). A ce stade du cycle biologique, le hapteur de la larve s'est avéré une structure intéressante, car son organisation est beaucoup plus simple. La voie était alors ouverte pour l'élaboration de nouvelles classifications. L'étude comparée du hapteur larvaire a permis, en effet, de définir des subdivisions mettant en évidence des affinités d'ordre évolutif, entre ces groupes.

Connaissant d'une part le hapteur de l'adulte et d'autre part celui de la larve libre, il était indispensable d'établir les rapports entre ces deux stades pour rechercher et comprendre les transformations liées à l'installation du parasite sur l'hôte. Baer et Euzet (1961), Llewellyn $(1963,1968)$ ont insisté sur ce nouvel aspect de la biologie des Monogènes ; mais l'effort des chercheurs a surtout porté sur les Polyopisthocotylea essentiellement branchiaux et hématophages. On connaît peu de choses sur les Monopisthocotylea qui sont des Monogènes cutanés ou branchiaux, mais qui se nourrissent dans les deux cas de cellules épithéliales et de mucus. 
Parmi ceux-ci, nous avons choisi d'étudier des Dactylogyridea. Malgré leur abondance chez les Téléostéens, ces Monopisthocotylea ont un développement mal connu. A l'origine cutanés, ils se sont secondairement diversifiés sur les branchies. Nos recherches sont axées sur la structure du hapteur larvaire et sur son évolution postlarvaire sur l'hôte. Elles s'inscrivent dans le cadre des problèmes posés par les modalités d'infestation et les potentialités adaptatives de l'organe de fixation, en fonction du substrat et du microbiotope.

Jusqu'à présent, le hapteur adulte ou larvaire a donc représenté le principal centre d'intérêt pour la connaissance des Monogènes. Les observations et quelques études expérimentales mènent à penser que le hapteur a été, au cours de l'évolution, extrêmement sensible aux phénomènes adaptatifs et que la sélection naturelle a eu sur lui un impact fondamental. Ainsi s'explique la grande diversité des systèmes de fixation par lesquels l'ectoparasite se maintient sur son hôte.

Pour mieux comprendre l'évolution des Monogènes, nous avons ressenti la nécessité d'étudier un autre système, moins spécialisé et moins sujet à variations, que le hapteur. Ce système devait satisfaire aux conditions suivantes:

- Etre le moins possible modifié par le parasitisme.

- Etre moins sensible que le hapteur à des phénomènes adaptatifs.

- Etre de mise en évidence commode.

Pour ces raisons, nous nous sommes adressés aux structures argyrophiles de l'oncomiracidium. En effet :

L'oncomiracidium est le stade libre qui représente chez les Monogènes parasites la seule phase de dispersion.

Le nitrate d'argent met en évidence, chez les Plathelminthes, les cellules ciliées locomotrices mais aussi, et surtout, les terminaisons sensorielles tégumentaires ou sensilles. Leur répartition est appelée chétotaxie. Son étude sur les cercaires de Trématodes (Richard, 1971; Bayssade-Dufour, 1978) a démontré sa valeur systématique et phylétique.

Cet appareil sensoriel, en relation avec le système nerveux, doit être, au moins chez la larve libre nageante, moins sujet que le hapteur à des variations d'ordre adaptatif.

Ces recherches sur les structures sensorielles et locomotrices de l'oncomiracidium ne se limitent pas aux aspects évolutifs discutés à partir de leur étude comparée, elles s'inscrivent dans le cadre des problèmes biologiques posés par la transmission parasitaire et par les modifications de la vie de relation liées au parasitisme.

Nous présentons ce travail sous la forme d'un article qui synthétise des résultats déjà publiés. Nous en donnons ci-dessous la liste complète.

1971 Compléments à l'étude de la larve de Diplozoon paradoxum Von Nordmann, 1832 (Monogenea). Ann. Parasitol. Hum. Comp., 46, 675-684 (avec L. Euzet).

1972 Evolution de l'appareil sensoriel au cours de la vie larvaire chez les Monogènes Polyopisthocotylea. C.R. Acad. Sci., 274, $402-404$ (avec C. Combes). 
1974 Chétotaxie comparée des larves de Diplozoon paradoxum Von Nordmann, 1832 et de Diplozoon gracile Reichenbach Klinke, 1961 (Monogenea). Bull. Soc. Zool. Fr., 99, 307-314 (avec L. Euzet).

1974 Parasitisme branchial simultané par deux espèces congénériques de Diplectanum Diesing, 1858 (Monogenea, Monopisthocotylea) chez Dicentrarchus labrax (L., 1758) (Téléostéen). C.R. Acad. Sci., 279, 1345-1347 (avec C. Maillard).

1975 La chétotaxie des larves nageantes de Polystomes européens (Monogenea). Ann. Parasitol. Hum. Comp., 50, 25-37 (avec C. Combes).

1975 Développement post-larvaire d'Actinocleidus sp. Mueller, 1937 (Monogenea, Monopisthocotylea, Ancyrocephalidae). Hypothèse de la migration des onchoblastes chez les Dactylogyroidea. C.R. Acad. Sci., 281, 1329-1332.

1975 Répartition branchiale de deux Monogènes: Diplectanum aequans et Diplectanum laubieri (Monogenea, Monopisthocotylea) parasites simultanés de Dicentrarchus labrax (Téléostéen). Ann. Parasitol. Hum. Comp., 50, $691-699$ (avec C. Maillard).

1976 Mise en évidence et importance de la chétotaxie larvaire chez les Monogènes Monopisthocotylea. C.R. Acad. Sci., 282, 1109-1112.

1977 Position systématique et biologie d'Ergenstrema mugilis Paperna, 1964 (Monogenea, Monopisthocotylea) parasite de Liza (Liza) ramada (Risso, 1826) (Téléostéen Mugilidae). Bull. Mus. Nat. Hist. Nat., $3^{\circ}$ sér., 472, Zool., 329, 823-831 (avec D. Sanfilippo).

1977 Développement larvaire et post-larvaire d'Ergenstrema mugilis Paperna, 1964 (Monogène Ancyrocephalidae) parasite de Liza ramada (Risso, 1826) (Téléostéen Mugilidae). Z. Parasitenk., 52, 229-240.

1977 L'oncomiracidium d'Ancyrocephalus paradoxus (Monogenea, Monopisthocotylea) parasite de Sander lucioperca (Téléostéen, Percidae). Ann. Parasitol. Hum. Comp., 52, 493-505.

1977 Recherches sur les affinités phylétiques des Polystomatidae. C.R. Acad. Sci., Sér. D, $285,1243-1246$.

1978 Précisions sur l'oncomiracidium de Tetraonchus monenteron (Wagener, 1857) Diesing 1858 (Monogenea, Tetraonchidae) parasite d'Esox lucius L. (Téléostéen). Ann. Parasitol. Hum. Comp., 53, 117-121.

1978 Chétotaxie larvaire et phylogénie des Monogènes. IV $V^{e}$ Congrès International de Parasitologie, 19-26 août 1978, Varsovie, Icopa IV.

1978 L'oncomiracidium de Eupolystoma alluaudi (De Beauchamp, 1913), Monogène parasite de Bufo regularis Reuss en Afrique. Bull. Na. Hist. Nat. Paris, 3 sér., 514, Zool., 353, 211-216 (avec C. Combes, R. Bourgat et M.-L. Salami-Cadoux).

1978 Morphologie de l'oncomiracidium de Polystomoides Ward, 1917 (Monogenea) et situation du genre parmi les Polystomatidae. Z. Parasitenk., 56, 175-181 (avec C. Combes et M. H. Ktari).

1978 Recherches sur l'oncomiracidium des Capsalidae (Monogenea). Ann. Parasitol. Hum. Comp., 53, 351-357.

1978 L'oncomiracidium de Metapolystoma brygoonis (Euzet et Combes, 1964) parasite de l'Amphibien malgache Ptychadena mascareniensis (Dumeril et Bribon). Ann. Parasitol. Hum. Comp., 53, 547-549 (avec R. Bourgat).

1978 Recherches sur les stades larvaires des Monogènes de Poissons. Ann. Parasitol. Hum. Comp., 53, 551-559.

1979 Recherches sur les affinités phylétiques des Vers parasites Gyrodactylidae (Monogenea). C.R. Acad. Sci., 288, 231-233. 
1979 Evolution post-larvaire de l'appareil sensoriel chez les Dactylogyridea (Monogenea). Z. Parasitenk., 58, 259-263.

1979 L'oncomiracidium d'Erpocotyle catenulata (Guberlet, 1933) (Monogène Hexabothriidae) parasite de Mustelus mustelus (L.) (Selachii). Ann. Parasitol. Hum. Comp., 54, 113-115 (avec C. Maillard).

Cette première partie comporte deux chapitres: le premier est consacré aux méthodes expérimentales utilisées; le second se rapporte à l'étude du développement post-larvaire chez les Dactylogyridea et présente deux aspects qui sont, successivement, les modalités d'infestation de l'hôte et la morphogénèse du hapteur.

La seconde partie (à paraître) sera consacrée à l'étude des structures argyrophiles des oncomiracidiums et leur implication dans la phylogénèse des Monogènes.

\section{Matériel et méthodes}

Les Monogènes représentent, parmi les Plathelminthes, un matériel de laboratoire difficile à étudier car ce sont essentiellement des ectoparasites de Poissons et leur spécificité vis-à-vis de leur hôte est généralement stricte. Par rapport aux Plathelminthes endoparasites, ils ne sont pas un matériel expérimental commode, car leur biologie reste encore mal connue : bien qu'holoxène, personne ne maîtrise actuellement, au laboratoire, le cyle complet des Monogènes de Poissons. On a peu d'informations sur l'influence des facteurs du milieu, à tous les niveaux du cycle : modalités d'infestation, croissance et reproduction. En outre, il est fréquent d'observer en aquarium des hyperinfestations, des défaunations spontanées ou le développement d'autres parasitoses (Protozoaires, Champignons).

Le développement de ces Helminthes est schématisé figure 1.

Tout travail sur les stades larvaires de ces parasites nécessite un matériel abondant et vivant. Les Poissons hôtes, d'où qu'ils viennent, doivent être examinés rapidement, 4 à 5 heures après leur capture. Au-delà, les Vers, très affaiblis, ne pondent plus, ou bien donnent des œufs tératologiques ou non fécondés. Cela nécessite un transport rapide du Poisson du lieu de récolte au laboratoire, dans de bonnes conditions.

\section{I. - Les Poissons hôtes.}

En milieu marin, le Poisson est acheté aux pêcheurs à la sortie du filet de capture. Nous distinguerons :

- La pêche au chalut : ce mode de pêche, s'il donne un matériel abondant et varié, présente très souvent l'inconvénient de fournir des échantillons qui n'ont plus les caractères de fraîcheur exigés pour obtenir des pontes abondantes.

- D'autres techniques de pêche permettent d'obtenir des Poissons vivants à leur sortie de l'eau : la senne de plage (dite "à la traîne ") procure des Poissons côtiers, mais elle est limitée dans le temps (de juin à septembre). La pêche au trémail («les 


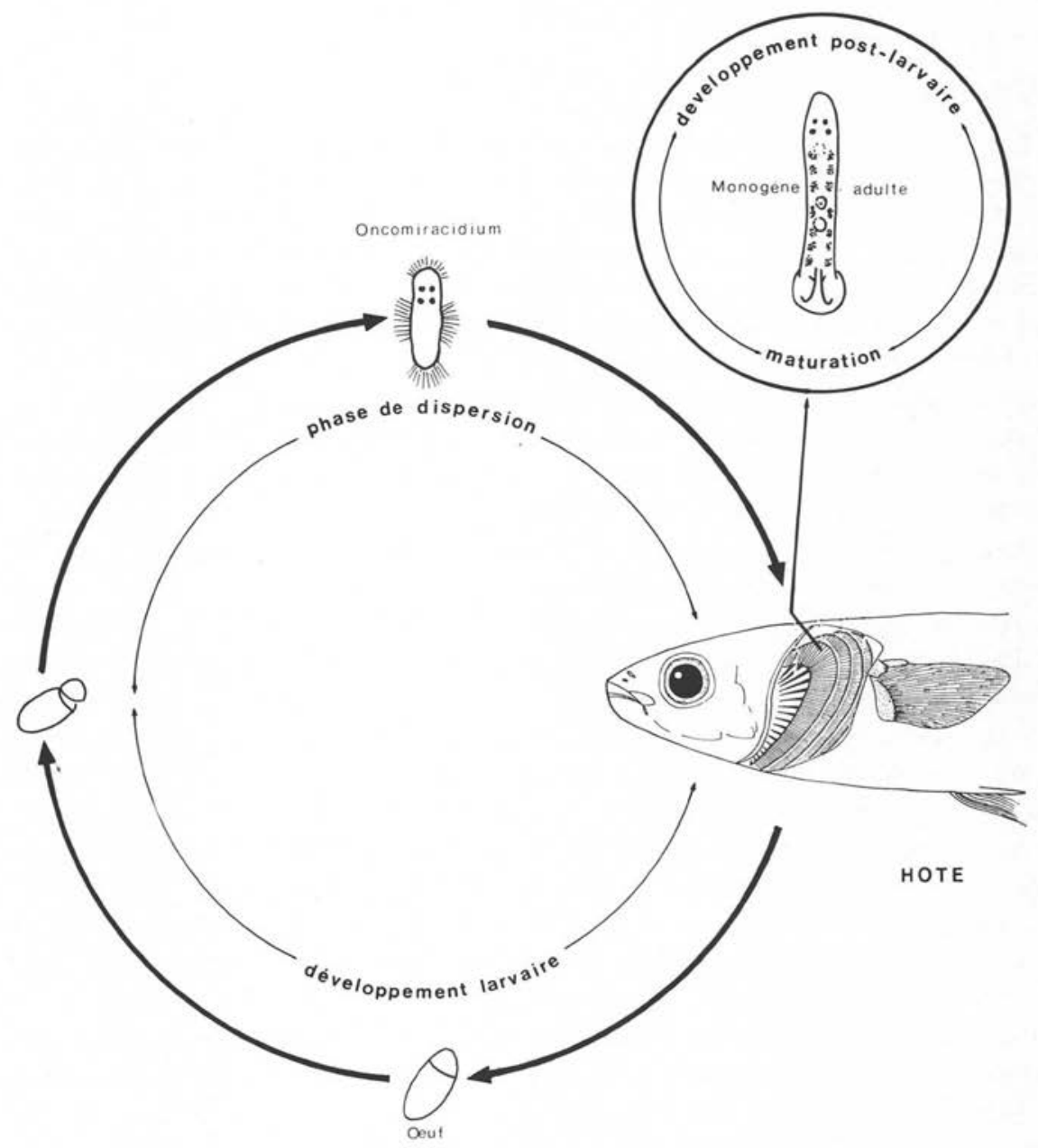

Fig. 1. Cycle biologique des Monogènes.

Life cycle of Monogenea.

pièces »), au filet fixe («capetchade »), au carrelet ("globe ») permet de récolter surtout les Téléostéens provenant des étangs littoraux.

En milieu dulcicole, la pêche professionnelle n'étant pas, à de rares exceptions près, représentée, nous avons utilisé la pêche électrique. Cette technique limite les prélèvements aux petites rivières de faible profondeur, mais elle a l'avantage de procurer du Poisson vivant. Le transport se fait dans des bacs où diffuse de l'oxygène pur $(0,1$ à $0,2 \mathrm{l} / \mathrm{mn})$. Les Poissons sont maintenus au laboratoire dans des bacs où l'eau, en circuit fermé, est filtrée selon deux méthodes : un filtrage rapide grâce à une pompe de type "Eheim » et un filtrage lent avec aérateur et laine de verre. 


\section{II. - Les parasites.}

\section{a. De l'adulte à l'oncomiracidium.}

Beaucoup de Monogènes occupent un microbiotope branchial. Dans la majorité des cas, dès la mort de l'hôte, il faut prélever les parasites sous la loupe binoculaire, directement sur les filaments branchiaux. Pour cela, on procède d'abord à l'ablation de l'opercule, puis on isole chaque arc branchial dans une coupelle contenant de l'eau (de mer ou de l'eau douce suivant le cas). Les Vers adultes, une fois détachés grâce à des aiguilles fines, sont pipetés et déposés dans des salières préalablement remplies d'eau filtrée (sur membrane "Millipore » à $0,22 \mu \mathrm{m}$ ).

Si les parasites ont été prélevés dans d'excellentes conditions de fraîcheur et s'ils sont mûrs, ils pondent rapidement une fois détachés de l'hôte. Il faut ensuite retirer de la salière (12 à 24 heures après le prélèvement) les adultes dont la survie, dans ces conditions, n'excède guère 24 heures.

Pour que les œufs se développent, il faut attendre plusieurs jours, la durée de l'incubation variant en fonction des espèces et de la température. Afin d'éviter ou de limiter au maximum les développements bactériens et fongiques, ou la prolifération de Ciliés, il est nécessaire de changer quotidiennement l'eau d'élevage par une eau filtrée sur « Millipore».

L'ontogénèse in ovo achevée, les oncomiracidiums s'échappent des œufs en soulevant un opercule qui laisse une ouverture circulaire dans la coque. La durée de vie de ces larves est brève dans les conditions d'observation (12 à $24 \mathrm{~h}$ ). Généralement attirées vers la surface par un phototropisme positif, beaucoup meurent plaquées sur le film de surface et au niveau du ménisque créé par les forces de tension superficielles.

Dans quelques cas, on peut obtenir les œufs des parasites sans procéder à la dissection de l'hôte, s'il est possible de le maintenir en vie et si le Ver pond des œufs suffisamment gros.

Cela est possible pour les Polystomes vésicaux d'Amphibiens, en isolant les Grenouilles dans de petits cristallisoirs; il suffit de pipeter quotidiennement les œufs émis dans l'eau avec l'urine (Combes, $1972 a$ ).

Pour les espèces du genre Diplozoon, parasites de Cyprinidae d'eau douce, nous nous sommes inspirés d'une technique utilisée par Bovet (1967) qui a utilisé le courant d'eau circulaire créé dans les bacs pour récolter, sur un tampon filtrant, les œufs des parasites. Nous avons utilisé une gaze de type moustiquaire, tendue sur un entonnoir de verre, branché sur le siphon inhalant d'une pompe filtrante de type "Eheim ». Les œufs de forte taille $(300 \mu \mathrm{m})$ munis d'un long filament sont retenus sur la gaze qu'il suffit de prélever. On procède ensuite au tri des œufs, sous la loupe binoculaire, afin de les isoler des autres détritus amassés sur le filtre.

\section{b. De l'oncomiracidium à la post-larve.}

Il semble y avoir, chez les Monogènes, deux modalités d'infestation de l'hôte par l'oncomiracidium : 
- Chez les Polyopisthocotylea, l'oncomiracidium gagne directement le microbiotope branchial par le courant d'eau respiratoire buccal.

- Chez les Monopisthocotylea, l'oncomiracidium se fixe d'abord sur le corps de l'hôte et gagne secondairement les branchies. Pour ces Monogènes, une phase cutanée s'intercale donc entre la phase libre et la phase branchiale caractéristique de l'adulte.

Dans les deux cas, pour récolter des post-larves, il est nécessaire d'avoir des hôtes vivants. Il y a deux manières d'obtenir ces stades expérimentalement :

- Si on dispose de Poissons vierges de parasites, on les met en présence, dans un petit aquarium muni d'un aérateur ou d'un circuit filtrant de type lent, avec des oncomiracidiums venant d'éclore.

- Si on dispose de Poissons parasités, il est possible, en les maintenant dans un bac en circuit fermé, d'obtenir des hyperinfestations, si l'eau est filtrée lentement.

Dans le cas des Polyopisthocotylea, les jeunes post-larves seront recherchées et récoltées sur les filaments branchiaux.

Dans le cas des Monopisthocotylea, la détection des post-larves sur le corps des Poissons est délicate. Il est donc nécessaire d'utiliser des hôtes expérimentaux de petite taille (3 à $6 \mathrm{~cm}$ ), ce qui permet d'explorer toute la surface du Poisson sous la loupe binoculaire. Après cet examen, on procède à l'ablation des opercules et des nageoires pour détecter les post-larves qui peuvent être sur la face interne du volet operculaire ou entre les rayons des nageoires. Les stades post-larvaires plus avancés dans le développement et les adultes sont recherchés sur l'appareil branchial.

\section{c. Etude des oncomiracidiums et des post-larves.}

Larves et post-larves sont étudiées sur le vivant, dans une goutte d'eau, entre lame et lamelle. C'est la seule méthode actuellement connue pour observer le système excréteur constitué de protonéphridies, de canalicules et de canaux collecteurs.

Le hapteur demande une attention toute particulière: chacune des pièces qui le constitue doit être examinée en détail. Il faut connaitre leur nombre, leur position, leur orientation (dorsale ou ventrale), ainsi que leur morphologie précise. Elles sont dessinées à la chambre claire Wild, montée sur un microscope Wild M 20. On a, dans le passé, utilisé diverses nomenclatures et au moins cinq systèmes de numérotation pour identifier les différentes pièces du hapteur.

La table ronde organisée au IV $\mathrm{V}^{\mathrm{e}}$ Congrès International de Parasitologie (Icopa IV) avait pour objectif d'élaborer un système unique de nomenclature et de numérotation des crochets. La numérotation proposée par Llewellyn (1963) a été adoptée ainsi que le terme de hamulus (pluriel : hamuli) pour désigner les grands crochets du hapteur des Dactylogyridea.

Chez les Dactylogyridae, on a convenu de nommer $\mathrm{R}$ les fines structures sclérifiées que Mizelle et Price (1963) dénommaient 4 A. Quant aux autres éléments du hapteur, il n'y a pas eu d'accord sur les termes à employer pour les désigner, l'homologie de ces pièces étant encore discutée. 
Le présent travail synthétisant des travaux antérieurs à cette table ronde, nous avons conservé la numérotation que nous avons auparavant adoptée et qui est celle de Euzet et Ktari (1970). Ceci pour éviter les confusions avec les travaux déjà publiés et préserver ainsi l'unité du travail.

Les crochetons sont numérotés d'arrière en avant, comme le montrent les figures 2 et 3 : d'après Llewellyn (2); numérotation utilisée dans ce travail (3).
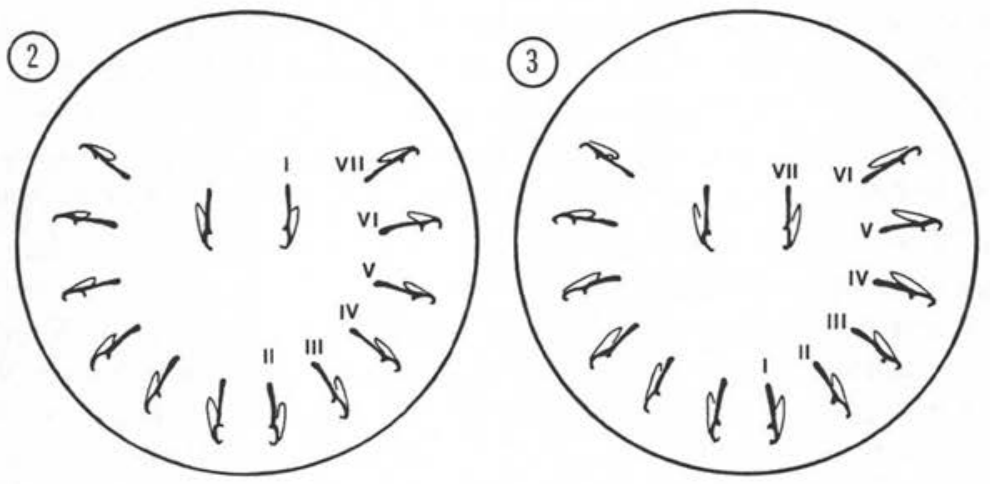

Fig. 2. Numérotation des crochets d'après Llewellyn. Hooklet numeration according to Llewellyn.

Fig. 3. Numérotation utilisée dans ce travail. Hooklet numeration used in the present study.

\section{III. - Les imprégnations argentiques.}

Nous entendons par "imprégnation argentique" la coloration in toto avec le nitrate d'argent de diverses structures des Monogènes.

\section{a. Les structures argyrophiles des Monogènes.}

Le nitrate d'argent révèle, chez les Monogènes, les terminaisons sensorielles ou sensilles et les cellules ciliées larvaires. Mais il marque aussi des orifices glandulaires divers au niveau de la région buccale et les pores excréteurs. Dans le hapteur, les zones, où les pointes des pièces sclérifiées émergent du tégument, s'imprègnent fortement rendant délicate l'interprétation de cette région.

Comme cela a été mis en évidence chez les Trématodes, en microscopie optique (Rohde, 1966, 1968, 1972) et en microscopie électronique (Dixon et Mercer, 1965 ; Erasmus, 1967 ; Morris et Threadgold, 1967 ; Wilson, 1970 ; Matricon-Gondran, 1971 ; Brooker, 1972), il existe aussi divers types de récepteurs sensoriels tégumentaires chez les Monogènes. Leur ultrastructure a été étudiée par Lyons (1969 $a$ et $b, 1972 a, 1973 a$ ). Elle distingue, photorécepteurs mis à part, les récepteurs simples uniciliés, les récepteurs complexes uniciliés, les récepteurs complexes multiciliés et les récepteurs en 
massue ou en bouton. L'imprégnation au nitrate d'argent n'étant pas spécifique, elle ne permet pas de distinguer les différents types de papilles sensorielles citées ci-dessus. Toutes se présentent (à l'exception sans doute des terminaisons en massue sous-tégumentaires) comme un anneau brun, généralement circulaire, parfois ovale, dont le diamètre est compris entre 1 et $2 \mu \mathrm{m}$. D'après l'ultrastructure des récepteurs, il semble que les desmosomes, qui ancrent les boutons sensoriels dans le syncytium tégumentaire, soient argyrophiles, d'où cet aspect en anneau. Les cellules ciliées sont imprégnées sur leur limitante périphérique.

Dans les régions buccale et haptoriale, il est souvent difficile de distinguer nettement les différentes formations argyrophiles : ces zones réagissent plus irrégulièrement que le reste du corps à l'imprégnation, peut-être à cause de rejets sécrétoires qui empêchent momentanément la réaction. Lorsque la réaction est positive, il est difficile de différencier avec certitude certains orifices glandulaires de sensilles multiciliées.

Le nitrate d'argent imprègne donc des structures hétérogènes et n'est pas un marqueur spécifique, au sens cytochimique; par contre, il a l'avantage de mettre en évidence, in toto, les sensilles et les cellules ciliées. C'est donc, jusqu'à présent, la seule technique qui permette l'étude précise de la chétotaxie et de l'appareil locomoteur larvaire. Les méthodes traditionnelles d'étude in vivo ne permettaient de décrire, outre la morphologie générale des oncomiracidiums, que l'armature du hapteur et la disposition du système excréteur. Or, le but de ce travail est précisément de démontrer l'importance fondamentale de ces nouvelles données morpho-anatomiques des stades larvaires. Il est souhaitable que toute nouvelle description d'un oncomiracidium ou d'une post-larve de Monogène comporte une étude des cellules ciliées et de la chétotaxie.

Par leur nombre et leur disposition constante, toujours symétrique par rapport au plan de symétrie bilatérale, les sensilles nous ont permis de mettre en évidence différents plans d'organisation de cet appareil sensoriel tégumentaire, plans qui caractérisent les grands groupes de Monogènes.

\section{b. Groupage et nomenclature des sensilles.}

N'ayant au départ aucun point de comparaison, nous avons adopté, comme Combes (1968), un « système de groupage basé sur les structures constantes observées, uniquement dans un but pratique ».

Nous avons utilisé un système de nomenclature pour les Monopisthocotylea et pour les Polyopisthocotylea (Lambert, 1977 c). Bien que la dénomination des groupes soit, dans le détail, légèrement différente pour chacune des deux sous-classes, c'est fondamentalement un système de groupage longitudinal des sensilles.

Cependant, nous avons essayé de mettre en place un système différent, inspiré de celui proposé par Richard (1971), pour les cercaires de Digènes (Combes et Lambert, 1975). Il diffère du précédent car c'est un groupage des sensilles en cycles transversaux. Nous l'avons volontairement limité aux Polystomatidae pour des raisons pratiques : cette famille est la seule qui a permis d'obtenir un matériel abondant et varié. 
Nous avons considéré, de ce fait, qu'elle avait valeur d'exemple pour tester un système de groupage sur la base de données plus générales.

Il sera nécessaire d'adopter un système unique pour nommer et grouper les sensilles chez tous les Monogènes. Nous ne pourrons le définir que lorsque nous disposerons d'un plus grand nombre d'oncomiracidiums connus.

La disposition des sensilles étant parfaitement symétrique, nous donnons, pour chaque groupe, leur nombre par hémicorps.

\section{c. Technique d'imprégnation en milieu d'eau douce.}

Pour les oncomiracidiums des Monogènes dulcicoles, nous avons utilisé une méthode directe, inspirée de celle de Lynch (1933), Croghan (1958), Ginetsinskaya et Dobrovolski (1963) et Lie (1966). Elle consiste à :

1. Projeter sur une larve encore vivante quelques gouttes d'une solution d'AgNO (3 à $8 \%)$.

2. Exposer la préparation: soit à la lumière solaire (5 à $10 \mathrm{mn})$, soit à la lumière artificielle (type lumière du jour) pendant $20 \mathrm{mn}$ environ. Cette exposition se fait à froid $\left(8-10^{\circ}\right)$.

3. Rincer abondamment plusieurs fois la préparation à l'eau distillée.

4. Monter :

- pour les larves de forte taille (ex. : Polystoma, Diplozoon), on effectue une déshydratation par passages successifs dans les alcools $70^{\circ}, 95^{\circ}, 100^{\circ}$ et butylique. Puis éclaircissement au Toluène et on monte au Baume du Canada.

- pour les larves de petite taille (type : Dactylogyridae), le montage s'effectue, sans déshydratation, après le rinçage, dans une gomme au chloral (type : liquide de Faure). Il faut alors luter la préparation (Combes, Bayssade-Dufour, Cassone, 1976).

\section{d. Technique d'imprégnation en milieu marin.}

En milieu marin ou saumâtre, l'utilisation du nitrate d'Argent est rendue délicate et difficile par la présence de chlorures dont la moindre trace provoque un précipité insoluble de chlorure d'Argent.

Les essais que nous avons tentés avec des techniques faisant intervenir une préfixation, ont été jusqu'ici négatifs (techniques de Chatton et Lwoff, 1930 ; Wagner, 1961 ; Richard, 1971). Finalement, nous avons obtenu des résultats avec la technique suivante :

1. Prélever une larve vivante et la déposer sur une lame très propre dans une goutte d'eau de mer, la plus petite possible.

2. Pipeter l'excès d'eau de mer et laisser tomber sur la larve plusieurs gouttes d'eau distillée.

3. Pipeter cette eau de rinçage au maximum et projeter sur la larve une solution d'AgNO $\mathrm{N}_{3}$ (6\% environ).

Ces manipulations doivent s'effectuer le plus rapidement possible car le nitrate d'Argent doit imprégner la larve encore vivante. 
4. Exposer la préparation,

5. Rincer,

6. Monter.

comme précédemment.

Cette méthode amène les commentaires et remarques suivants :

- Pour avoir une imprégnation positive, il nous est apparu indispensable que la larve soit encore vivante lorsqu'on projette sur elle la solution de nitrate d'Argent. Les larves marines sont évidemment tuées par l'eau distillée ; il faut donc manipuler très rapidement pour, successivement, éliminer l'eau de mer par rinçage et procéder à l'imprégnation avant la mort de l'oncomiracidium.

Deux risques d'échec découlent de tout ceci :

- Bien que très rapide, le rinçage doit être efficace car, s'il reste quelques traces de chlorures, un précipité de $\mathrm{AgCl}$ se produit. C'est pour cette rasion que nous manipulons les larves, une par une, en les déposant dans une goutte d'eau de mer qui doit être la plus petite possible.

- Les oncomiracidiums de certaines espèces sont tués très rapidement par l'eau distillée alors que d'autres résistent mieux comme c'est souvent le cas des Monogènes de Poissons plus euryhalins. Ceci a contribué à resserrer l'éventail des larves décrites. Ainsi, malgré un abondant matériel, nous n'avons jamais eu de préparation positive avec les oncomiracidiums de Kuhnia scombri, parasite de Scomber scombrus L.

- Conservation des préparations : les préparations peuvent être conservées très longtemps si on effectue la déshydratation par les alcools, l'éclaircissement par le Toluène et le montage au Baume du Canada. Les larves jaunissent un peu, mais le contraste persistera entre les sensilles (ou les cellules ciliées) et le reste du corps.

Par contre, le montage direct dans la gomme au chloral, ne donne pas de préparations permanentes. Il faut les étudier et les dessiner à la chambre claire dans les jours qui suivent le montage. Néanmoins, l'avantage de cette méthode par rapport à la précédente est qu'elle limite au maximum les manipulations et évite ainsi la perte de larves lorsque l'oncomiracidium est de petite taille ou le matériel en petite quantité.

Nous donnons ci-dessous, la liste des espèces étudiées dans ce travail.

\section{ANCYROCEPHALIDAE.}

- Actinocleidus oculatus (Mueller, 1934).

Lepomis gibbosus (L.) (S.E. France), Lambert, 1975.

- Actinocleidus recurvatus Mizelle et Donahue, 1944.

Lepomis gibbosus (L.) (S.E. France), Lambert, 1975 et 1979 b.

- Ancyrocephalus paradoxus Creplin, 1839.

Stizostedion lucioperca (L.) (S.E. France), Lambert, 1977 b.

- Ergenstrema mugilis Paperna, 1964.

Liza ramada (Risso, 1826) (S.E. France), Lambert, 1976, 1977 a ; Lambert et Sanfilippo, 1977.

- Ligophorus vanbenedenii (Parona et Perugia, 1890).

Liza aurata (Risso, 1810) (S.E. France), Lambert, $1978 d$. 
- Annulotrema sp. Alestes nurse Ruppel, 1832 (Tchad), Lambert, présent travail.

- Onchocleidus principalis (Mizelle, 1936).

Micropterus salmoïdes (Lacépède, 1802) (S.E. France), Lambert, $1978 d$ et présent travail.

Calceostomatidae.

- Calceostomella inerme (Parona et Perugia, 1889).

Umbrina cirrhosa (L.) (Tunisie, Tunis), Lambert, $1978 d$.

CAPSALIDAE.

- Benedenia monticelli (Parona et Perugia, 1895). Mugil cephalus L. (S.E. France), Lambert, 1978 c.

- Entobdella soleae (Van Beneden et Hesse, 1863). Solea vulgaris Quensel, 1806 (France, Brest), Lambert, 1978 c.

- Trochopus pini (Van Beneden et Hesse, 1863). Trigla lucerna L. (S.E. France), Lambert, 1978 c.

\section{DACTYLOGYRIDAE.}

- Dactylogyrus extensus (Mueller et Van Cleave, 1932). Cyprinus carpio L. (S.E. France), Lambert, 1976, 1977 b, 1979 b et présent travail.

- Neodactylogyrus wunderi (Bychowski, 1931). Abramis brama (L.) (S.E. France), Lambert, présent travail.

DiPLECTANIDAE.

- Diplectanum aequans (Wagener, 1857). Dicentrarchus labrax (L.) (S.E. France). Lambert et Maillard, 1975 ; Lambert, 1977 b, $c$ et présent travail.

- Diplectanum laubieri Lambert et Maillard, 1974. Dicentrarchus labrax (L.) (S.E. France), Lambert et Maillard, 1974, 1975.

- Furnestinia echeneis (Wagener, 1857). Sparus aurata L. (S.E. France), Lambert, $1978 d$.

MONOCOTYLIDAE.

- Calicotyle kröyeri Diesing, 1850.

Raja clavata L. (S.E. France), Lambert, $1978 d$.

TETRAONCHIDAE.

- Tetraonchus monenteron (Wagener, 1857).

Esox lucius L. (S.E. France), Lambert, $1978 a$.

UDONELLIDAE.

- Udonella caligorum Johnston, 1835.

Caligus minimus Otto, 1848 [Copépode de Dicentrarchus labrax (L.)], (S.E. France), Lambert, $1978 b$ et présent travail.

GyrodactyLidae.

- Gyrodactylus SP. 1. Carassius auratus (L.) (S.E. France), Lambert, 1979 a.

- Gyrodactylus SP. 2.

Phoxinus phoxinus (L.) (S.E. France), Lambert, présent travail. 


\section{DICLIDOPHORIDAE.}

- Cyclocotyla bellones (Otto. 1821).

Isopodes Symothoides de Boops boops L. et Spicara maena (L.) (S.E. France), Lamhert. $1978 d$.

\section{Discocotylidae.}

- Diplozoon gracile Reichenbach-Klinke, 1961.

Barbus meridionalis Risso, 1826 (S.E. France).

Chondrostoma taxostoma (Vallot, 1836).

Gobio gobio (L.).

Phoxinus phoxinus (L.).

Telestes soufia Risso, 1826, Euzet et Lambert, 1971 et 1974 ; Combes et Lambert, 1972.

- Diplozoon paradoxum Van Nordmann, 1832.

Abramis brama (L.) (S.E. France ; Suisse, Neuchâtel), Euzet et Lambert, 1974.

\section{GastrocotylidaE.}

- Gastrocotyle trachurii Van Beneden et Hesse, 1863.

Trachurus trachurus (L.) (S.E. France), Lambert, $1978 d$.

\section{HeXABOTHRIIDAE.}

- Erpocotyle catenulata (Guberlet, 1933).

Mustelus mustelus (L.) (Tunisie, Sfax), Lambert et Maillard, 1979.

- Epicotyle torpedinis (Price, 1942).

Torpedo marmorata Risso, 1810 (S.E. France), Lambert, présent travail.

\section{MAZocraeidae.}

- Mazocraes sp. (1).

Sardinella maderensis (Lowe, 1841) (Tunisie, Tunis), Lambert, $1978 d$.

\section{MicrocotyLidAE.}

- Microcotyle chrysophrii Van Beneden et Hesse, 1863.

Sparus aurata L. (S.E. France), Lambert, présent travail.

- Microcotyle mugilis Vogt, 1878.

Mugil cephalus L. (S.E. France), Lambert, présent travail.

- Microcotyle mormyri Lorenz, 1878.

Lithognathus mormyrus (L.) (S.E. France), Lambert, 1977 c.

- Polylabris tubicirrus (Paperna et Kohn, 1964).

Diplodus sargus (L.) (S.E. France).

D. annularis (L.).

D. vulgaris (Geoffroy St-Hilaire, 1817), Lambert, présent travail.

\section{Polystomatidae.}

- Eupolystoma alluaudi (De Beauchamp, 1913).

Bufo regulatis, Reuss (Togo), Combes, Lambert, Bourgat et Salami-Cadoux, 1978.

- Metapolystoma brygoonis (Euzet et Combes, 1964).

Ptychadena mascareniensis (Dumeril et Bribon) (Madagascar), Lambert et Bourgat, 1978.

- Polystoma integerrimum (Fröhlich, 1798).

Rana temporaria L. (S.E. France), Combes et Lambert, 1972, 1975.

(1) Ce Monogène est une nouvelle espèce qui reste à décrire. 
- Polystoma pelobatis (Euzet et Combes, 1966).

Pelobates cultripes (Cuvier) (S.E. France), Combes et Lambert, 1975 ; Lambert, présent travail.

- Polystoma gallieni Price, 1938.

Hyla meridionalis Boettg. (S.E. France), Combes et Lambert, 1975.

- Polystomoïdes ocellatum (Rudolphi, 1819) (2).

Clemis caspica var. leprosa (Tunisie, Tunis), Lambert, Combes et Ktari, 1978.

Nous avons étudié 38 espèces qui représentent 16 familles importantes. Certaines familles (exotiques ou non représentées en Méditerranée) n'ont pas pu être étudiées comme les Microbothriidae, les Acanthocotylidae, les Chimaericolidae..., mais cet échantillonnage est suffisamment représentatif des Monogènes.

\section{Développement post-larvaire et morphogenèse du hapteur chez les Dactylogyridea (Monogenea, Monopisthocotylea)}

Après plusieurs décennies consacrées surtout à un inventaire faunistique et à l'étude morpho-anatomique des Monogènes adultes, plusieurs auteurs s'engageaient presque simultanément dans de nouvelles voies de recherches sur ces parasites: Bychowsky (1957); Euzet $(1955,1957,1958)$; Llewellyn (1957 $a$ et $b, 1963)$. Tous ont montré la nécessité d'étudier la phase larvaire libre du cycle biologique. Pendant cette seconde période de recherche, les efforts ont porté sur le développement de ces Plathelminthes.

En fait, le pourcentage de larves ou de développements post-larvaires étudiés, est très faible par rapport au nombre d'espèces décrites. Les Polyopisthocotylea sont les mieux connus (Ktari, 1971), mais il reste encore de nombreuses lacunes à combler. Chez les Monopisthocotylea, les Capsalidae, les Acanthocotylidae et les Monocotylidae ont été étudiés par Kearn (1963 $a, 1967,1968 a)$.

Chez les Dactylogyridea, qui représentent l'ordre où les familles sont les plus diversifiées, nos connaissances étaient insuffisantes pour comprendre les modalités d'infestation et la morphogénèse du hapteur. Les résultats importants sont ceux de Bychowsky (1957), Prost (1963) et Kearn (1968 b) chez les Dactylogyridae, ceux de Kearn (1968 $b$ ) chez les Diplectanidae et Tetraonchidae, et ceux de Euzet et Ktari $(1970,1973)$ chez les Calceostomatidae.

Ces observations ont mis en évidence deux problèmes fondamentaux que nous envisagerons successivement dans ce chapitre.

- Le premier, d'ordre biologique, est relatif aux modalités d'infestation du Poisson-hôte par les oncomiracidiums. C'est la question de la période d'invasion entre la

(2) Cette attribution spécifique a été faite sous réserve d'une comparaison entre le matériel tunisien et les parasites d'Emys orbicularis d'Italie et d'Europe. 
phase libre nageante, qui assure la dispersion, et l'installation dans le microbiotope définitif.

- Le second, d'ordre morphogénétique, intéresse le hapteur et présente deux aspects. Il s'agit :

- d'une part, de l'origine embryologique des diverses pièces haptoriales qui composent l'organe de fixation,

- d'autre part, de la morphogénèse du hapteur au cours de l'installation du parasite sur l'hôte.

Nous insisterons sur l'influence du substrat et du mode d'attachement dans ces transformations adaptatives.

\section{Modalités d'infestation et installation du parasite.}

Les modalités d'infestation du Poisson-hôte par les oncomiracidiums nageants sont longtemps restées hypothétiques. Dès 1929, Wunder envisageait trois possibilités :

- Les œufs pondus restent fixés aux branchies et, à l'éclosion, la larve se trouve en contact direct avec cet organe.

- La larve se fixe d'abord sur le peau du Poisson-hôte puis migre secondairement vers les branchies.

- L'éclosion a lieu dans le milieu extérieur et les larves atteignent les branchies avec le courant d'eau respiratoire.

Il y a peu d'arguments en faveur de la première modalité. Deux cas seulement nous paraissent relever vraiment d'un tel processus : Bychowsky (1957) décrit le solide attachement des œufs de Nitzschia sturionis aux branchies des Esturgeons. Les œufs de Dionchus agassizi Goto, 1899 et de Dionchus remorae (McCallum, 1916) sont en grappe et restent sur les filaments branchiaux de l'hôte Echeneis naucrates. A l'éclosion, les larves, non ciliées, se fixent directement sur la branchie (Ktari, 1975, 1977).

Si quelques auteurs ont envisagé un rôle des filaments de l'œuf pour leur rétention sur l'hôte, nous pensons comme Llewellyn (1972) que ce n'est pas une modalité à généraliser. Ce système, d'une part, n'assure pas la dispersion du parasite, et, d'autre part, favorise la réinfestation de l'hôte ce qui se traduit par une hyperinfestation (effectivement observée par Ktari, 1977, chez les Dionchus).

Certains auteurs ont soupçonné une voie d'infestation cutanée pour les Dactylogyrus de la Carpe (Bychowsky, 1933 ; Gröben, 1940 ; Lajman, 1946 ; Bauer et Nikolskaya, 1954). Llewellyn $(1957 b)$ a admis cette hypothèse pour la plupart des Monogènes branchiaux. Les premières données précises sont dues à Prost (1963) qui a expérimenté avec de très jeunes Carpes. Cet auteur prouve que les oncomiracidiums nageants de Dactylogyrus anchoratus, sortis d'œufs isolés, se fixent d'abord sur la peau de l'hôte puis rampent vers les branchies. Kearn $(1968 b)$ apporte des preuves supplémentaires à ces observations, en montrant que les larves ciliées de Neodactylogyrus crucifer (Dactylogyridae), Diplectanum aequans (Diplectanidae) et Tetraonchus monenteron 
(Tetraonchidae) établissent un contact initial avec la peau du Poisson-hôte. Cela peut être la peau de toute la surface externe ou celle qui tapisse la cavité bucco-pharyngienne. Cependant, cette voie cutanée, mise en évidence chez les espèces citées cidessus, n'est pas considérée par tous les auteurs comme quantitativement importante. Iziumova (1956) et Paperna (1963), travaillant sur Dactylogyrus vastator, estiment que l'infestation des branchies de la Carpe se réalise surtout par les larves amenées passivement par le courant d'eau respiratoire. Les quelques post-larves cutanées observées, ne seraient pas toutes infestantes et ne représenteraient qu'une modalité secondaire de l'infestation.

Gussev et Kulemina (1971) pensent que la voie cutanée est limitée aux très jeunes Poissons et qu'au-delà d'un certain âge, l'infestation se fait par voie buccale.

$\mathrm{Au}$ cours de nos recherches sur le développement post-larvaire des Monogènes Dactylogyridea, nous avons pu observer de nombreuses post-larves très actives sur le corps des Poissons, hyperinfestés expérimentalement au laboratoire. Il s'agit de deux Actinocleidus: A. oculatus et A. recurvatus, parasites de Lepomis gibbosus (Lambert, 1975 ) et d'Ergenstrema mugilis parasite de Liza ramada (Lambert, 1977 a). Les postlarves (oncomiracidium après fixation, ou stade plus avancé) sont toujours observées sur la région antérieure de l'hôte (nageoires pelviennes, pectorales, région céphalique et opercule) alors que les pré-adultes sont toujours récoltés sur les branchies, avec les adultes.

Des frottis cutanés, d'une Carpe de forte taille $(43 \mathrm{~cm})$ laissée en circuit fermé pendant 10 jours (du 25-10-1974 au 5-11-1974), nous ont permis de récolter deux très jeunes post-larves de Dactylogyrus extensus (à l'autopsie, l'infestation branchiale s'est révélée être monospécifique). Ces post-larves possédaient les ébauches des hamuli dans la région postérieure du corps, en avant du hapteur. Ces ébauches mesuraient respectivement 3 et $12 \mu \mathrm{m}$. Les stades post-larvaires plus avancés ont tous été récoltés sur les branchies.

Des observations similaires ont été faites avec Diplectanum aequans. Deux jeunes Dicentrarchus labrax (3) de 8 et $10 \mathrm{~cm}$, sont mis en présence (le 6-5-1975) avec des larves de $D$. aequans venant d'éclore. Le premier individu examiné le 7-5-1975, présentait 3 post-larves sur le corps et aucune sur les branchies. Le second individu est sacrifié le 9-5-1975; 4 post-larves sont observées sur le corps (nageoires et tête), d'autres (14), à un stade de développement plus avancé, sont déjà sur les branchies.

Nous avons mis en évidence chez Dicentrarchus labrax, dans les conditions naturelles, un parasitisme branchial simultané par 2 espèces congénériques (Lambert et Maillard, 1974). Il existe une répartition branchiale différentielle pour ces deux espèces : Diplectanum aequans et $D$. laubieri, qui est aussi fonction du stade ontogénique des individus (ce phénomène a été confirmé par Oliver, 1976). A la suite de ces observations (Lambert et Maillard, 1975), il apparaît qu'une migration continue de la post-larve se produit depuis le contact cutané initial jusqu'au microhabitat branchial

(3) Les Poissons ont été élevées à partir de l'œuf et isolés de toute contamination naturelle. Nous remercions M. Barnabé qui nous les a procurés. 
de l'adulte. Toute se passe comme si l'invasion parasitaire s'effectuait par les racines des arcs jusqu'à la base des filaments branchiaux ; puis les parasites atteignent le microhabitat définitif par une seconde migration centrifuge, de l'arc branchial vers l'extrémité des filaments (fig. 4). Le développement des Diplectanum est progressif au cours

Dactylogyridea parasites branchisus.

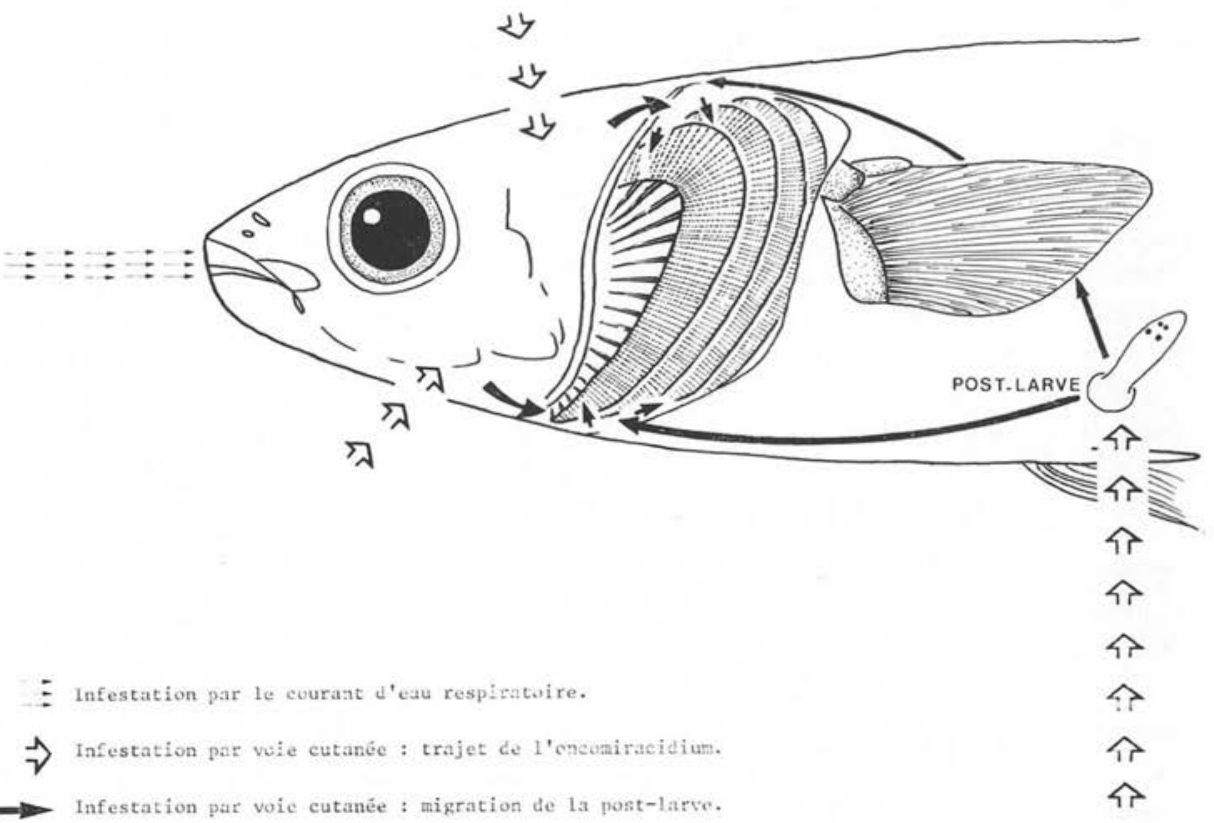

Fig. 4. Les voies d'invasion du Poisson hôte par les Dactylogyridea parasites branchiaux.

Routes of infestation of the fish host by Dactylogyridea gill parasites.

de cette migration continue (cutanée puis branchiale). La vitesse de développement des deux espèces n'est pas identique et permet d'expliquer leur répartition branchiale différente dans les conditions naturelles.

Une grande partie de nos observations confirment l'opinion de Kearn (1968 $b$ ) qui considère la fixation primaire de l'oncomiracidium sur le corps de l'hôte, comme une preuve de l'origine cutanée de ces parasites. La migration secondaire de la post-larve vers le microhabitat définitif de l'adulte, n'est pas l'apanage des seuls Monogènes branchiaux. Kearn $(1963 b)$ a montré que l'oncomiracidium d'Entobdella soleae (parasite cutané de Solea solea) infeste son hôte par la face zénithale, puis migre secondairement jusqu'à la face nadirale, microhabitat définitif de l'adulte. 
Cependant, toutes nos observations ne sont pas en accord avec les modalités d'infestation du Poisson-hôte exposées ci-dessus. Ainsi, nous n'avons trouvé les stades du développement post-larvaire d'Onchocleidus principalis que sur les branchies de Micropterus salmoides : un Black bass (M. salmoides) de $13 \mathrm{~cm}$, récolté dans la nature le 25-10-74, a été isolé en circuit fermé et autopsié 17 jours après, le 12-11-74. L'examen direct de la surface corporelle, ainsi que les frottis cutanés, n'ont livré aucune postlarve. Par contre, les branchies étaient recouvertes de nombreux individus à tous les stades du développement. Cette observation n'a pas été confirmée mais il est possible que, dans ce cas, il y ait eu infestation massive par voie buccale, ce qui confirmerait les observations d'Iziumova (1956) et Paperna (1963).

\section{En conclusion :}

- Chez les Polyopisthocotylea, il semble que les oncomiracidiums pénètrent passivement dans la chambre branchiale des hôtes, avec le courant d'eau respiratoire (Bovet, 1967 ; Paling, 1969 et Llewellyn, 1972). Par contre, des phénomènes de migration cutanée existent chez les Polystomatidae (Combes, 1968) et l'infestation à partir d'un " hôte intermédiaire » a été évoquée chez deux Gastrocotylidae (Bychowsky et Nagibina, 1967). L'existence de larves particulières (non nageantes, assymétriques, etc...) (Llewellyn, 1968 et Ktari, 1971) n'exclut pas d'autres modalités d'infestation adaptées à la biologie et à l'écologie de l'hôte.

- Chez les Monopisthocotylea : il existe, chez plusieurs espèces, la preuve d'un contact initial de l'oncomiracidium avec la peau du corps du Poisson-hôte, suivi d'une migration secondaire de la post-larve vers le microhabitat définitif branchial (Prost, 1963 ; Kearn, 1968 b ; Lambert, 1975, $1977 a$ ). Beaucoup de Monopisthocotylea sont d'ailleurs, à l'état adulte, des parasites de la peau et c'est pour ces deux raisons que nous pensons, comme Kearn (1968 b), que ces Monogènes sont, à l'origine, des parasites cutanés. Cependant, il paraît hasardeux, dans l'état actuel des connaissances, de généraliser le phénomène de l'infestation par le tégument. La détection des post-larves sur le corps des Poissons est difficile et aléatoire, et les techniques (observation directe et frottis cutanés) ne permettent pas d'avoir des données quantitatives. Avec les résultats actuels, uniquement qualitatifs, nous ne pouvons pas estimer l'importance relative de l'infestation branchiale par voie cutanée et par voie buccale. Cette dernière éventualité n'est pas à exclure et il faudra en estimer l'importance dans un bilan démographique du cycle parasitaire. Il reste aussi à préciser le rôle des facteurs biotiques et abiotiques qui influent sur les modalités d'infestation.

\section{Morphogénèse du hapteur.}

Depuis Bychowsky (1957), quelques larves de Monogènes Monopisthocotylea ont été décrites mais on ne connaît pratiquement rien sur le développement du hapteur larvaire et la manière dont il se transforme pour donner celui de l'adulte. Or, il existe 
une forte disproportion entre l'extrême variété morphologique du hapteur chez les adultes et la simplicité de cet organe chez les larves. Cette disproportion s'accroît considérablement si l'on ne considère pas seulement les types structuraux qui caractérisent les familles (par exemple, le type Dactylogyridae avec une paire de hamuli, le type Diplectanidae avec deux paires de hamuli et des squamodisques, etc...), mais si l'on tient compte des modes d'attachement qui varient à l'intérieur même de ces familles. Ainsi, Gussev (1976), qui a étudié les systèmes de fixation de nombreuses espèces, distingue dans le seul genre Dactylogyrus plusieurs types d'attachement (« wunderoid ", « anchoratoid », «sphyrnoid », etc...).

Comme nous l'avons vu, l'oncomiracidium peut se fixer et se déplacer sur le corps du Poisson-hôte. A ce stade du développement, cette larve possède un hapteur circulaire ventral parfaitement adapté à ce mode de fixation cutanée superficielle. Il est en forme de coupe aplatie qui joue comme une ventouse et dont l'adhésion à la peau de l'hôte est aidée par l'existence, à la périphérie du disque, de crochetons à ogive, tous orientés ventralement. Cet organe va se modifier, au cours du développement post-larvaire, pour s'adapter à une fixation branchiale caractérisée essentiellement par un accrochage entre les lamelles. La fixation ne se réalise pas seulement par des structures ventrales, mais aussi par la face dorsale du hapteur. Si, traditionnellement, on ne parle de métamorphose que chez les Polyopisthocotylea dont le hapteur larvaire est remplacé par des pinces ou des ventouses, on peut penser qu'il existe aussi une «métamorphose » chez les Dactylogyridea. Kearn (1968 b), le premier, a démontré le détail des transformations chez Diplectanum aequans et Neodactylogyrus crucifer.

Chez les Dactylogyridea, ces transformations de l'organe de fixation sont marquées :

1. par l'apparition de nouvelles structures: les hamuli,

2. par la nouvelle orientation (dorsale) que prennent certains crochetons larvaires.

Nous envisagerons successivement ces deux aspects de la morphogénèse du hapteur de l'adulte.

a. Origine des hamuli.

Généralement, l'oncomiracidium possède un hapteur armé uniquement par des crochetons périphériques. L'adulte possède en plus des grands crochets, les hamuli, dont le rôle est prépondérant dans l'attachement. Quelle est l'origine embryologique de ces pièces?

Kearn $(1968 b)$ a démontré l'origine corporelle des hamuli ventraux de Diplectanum aequans et des hamuli dorsaux de Neodactylogyrus crucifer. Chez les postlarves de Tetraonchus monenteron, les hamuli dorsaux et ventraux apparaissent dans la région postérieure du corps, en avant du hapteur. Par contre, il observe l'origine et la différenciation, dans le hapteur, des hamuli dorsaux de Diplectanum aequans.

Euzet et Ktari (1973) décrivent, chez les Calceostomatidae, des phénomènes analogues pour les hamuli dorsaux, alors qu'à l'éclosion les ventraux sont déjà formés dans le hapteur larvaire. 
Ces quelques observations ne permettent pas une compréhension globale de l'origine des hamuli et de leur mise en place, car, à part chez Tetraonchus monenteron, ces auteurs n'avaient observé que la migration d'une seule paire de hamuli (ventraux chez les Diplectanidae, dorsaux chez les Dactylogyridae et Calceostomatidae). Or, ces parasites présentent un hapteur quelque peu original par rapport à celui d'autres Monogènes mieux représentés chez les Téléostéens. Les Diplectanidae possèdent en avant du hapteur des structures accessoires d'attachement: les squamodisques. Les Dactylogyridae ont une seule paire de hamuli (les dorsaux) fonctionnels. Les Calceostomatidae ont un hapteur très spécialisé.

Nous avons démontré l'origine corporelle des hamuli dorsaux et ventraux et l'existence d'une migration lors de leur mise en place dans le hapteur, chez les quatre espèces que nous avons étudiées. Celles-ci appartiennent toutes à la famille des Ancyrocephalidae; ce sont: Actinocleidus recurvatus et A. oculatus (Lambert, 1975), Ergenstrema mugilis (Lambert, 1977 a) et Onchocleidus principalis (présent travail).

Nous avons suivi pour chacune la migration des hamuli, depuis leur origine corporelle où ils sont à l'état d'ébauches jusqu'à leur mise en place dans le hapteur fonctionnel.

D'autres observations confirment ces résultats expérimentaux : nous avons observé sur Alestes nurse une post-larve d'Annulotrema avec les ébauches des hamuli ventraux en avant du hapteur. Il en est de même chez la larve d'Ancyrocephalus paradoxus (Lambert, 1977 b) et chez celles de trois espèces d'Ancylodiscoides (Bychowsky, 1957).

Nos observations, s'ajoutant aux connaissances acquises précédemment, permettent d'émettre l'idée qu'il existe une migration généralisée des onchoblastes chez les Dactylogyridea (Lambert, 1975). Dans cette hypothèse, nous admettons que la migration affecte les onchoblastes (cellules responsables de la formation des hamuli), que ces cellules soient ou non en activité. Cette hypothèse implique l'origine corporelle de ces onchoblastes, donc de tous les hamuli, qu'ils soient dorsaux ou ventraux. Dans le cas de la migration des onchoblastes en activité, on observe effectivement cette migration grâce aux scléroprotéines des hamuli en formation. Dans le cas de la migration des onchoblastes inactifs, les hamuli semblent apparaître dans le hapteur. Dans cette dernière éventualité, on peut expliquer l'origine apparemment haptoriale des hamuli dorsaux de Diplectanum aequans, des hamuli ventraux des Calceostomatidae et des ébauches avortives ventrales des Dactylogyridae. Ainsi, lorsqu'une paire de hamuli existe dans le hapteur larvaire, à l'éclosion (ex. : Calceostomatidae), la migration aurait lieu lors du développement de l'embryon dans l'œuf. Ceci reste à démontrer, mais cette hypothèse permet d'expliquer tous les cas connus (fig. 5).

Cette migration peut être plus ou moins synchrone entre les hamuli ventraux et les hamuli dorsaux. Chez Actinocleidus, les hamuli ventraux apparaissent les premiers et migrent jusqu'à leur position définitive, puis ce sont les dorsaux. On peut cependant observer, chez certaines post-larves, les premières ébauches des hamuli dorsaux alors que les ventraux ne sont pas tout à fait à l'extrémité postérieure du hapteur. Chez Ergenstrema mugilis, nous avons montré qu'il existait des variations individuelles dans le synchronisme de la migration des hamuli (Lambert, 1977 a). 


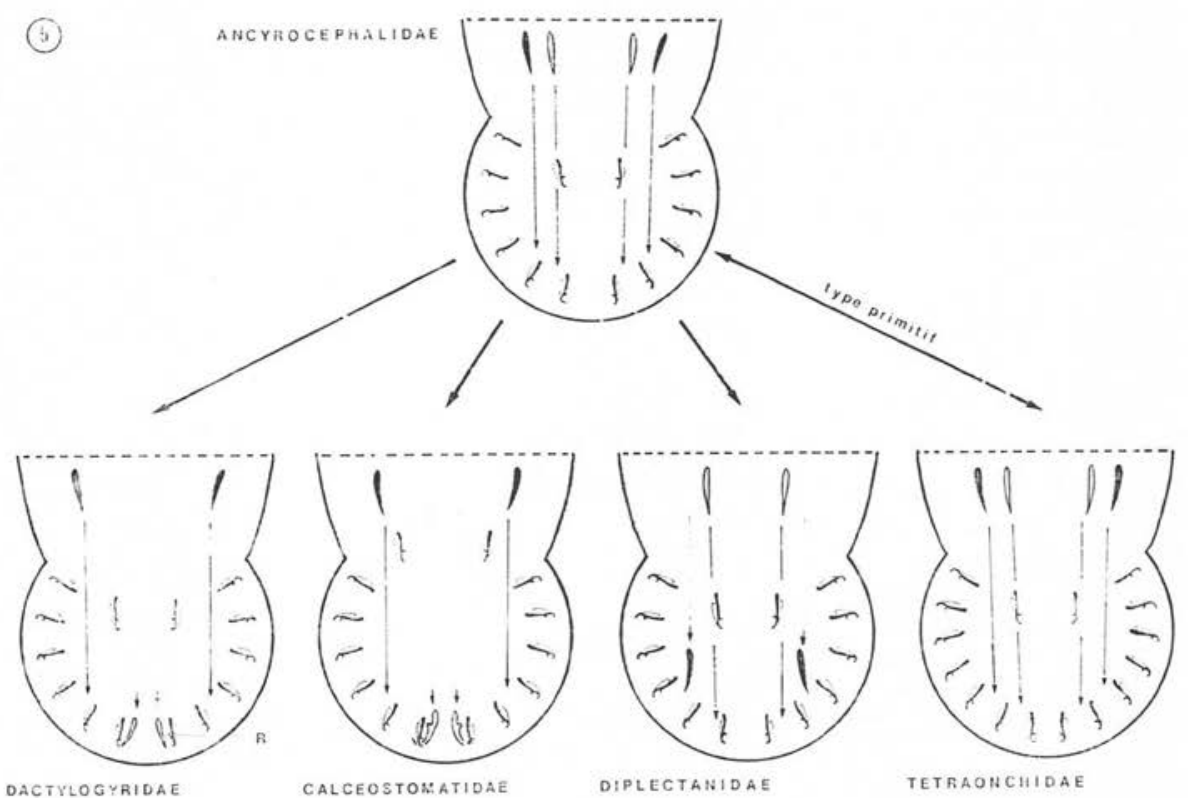

Fig. 5. Hypothèse de la migration des onchoblastes chez les Dactylogyridea. En blanc: ébauches des hamuli ventraux; en noir: ébauches des hamuli dorsaux; en trait plein : migration des onchoblastes en activité ; en pointillé : migration des onchoblastes inactifs. $\mathrm{R}$ : structure " $\mathrm{R}$ » des Dactylogyridae considérée comme l'homologue des hamuli ventraux.

Hypothesis on migration of onchoblasts in Dactylogyridea. In white: ventral hamu'i rudiments; in black : dorsal hamuli rudiments ; in continous line : migration of onchoblasts in activity; in broken line : migration of inactive onchoblasts. $\mathrm{R}$ : " $\mathrm{R}$ " structure of Dactylogyridea corresponding to ventral hamuli.

Par contre, chez Onchocleidus principalis, la migration et la morphogénèse des hamuli sont concomitantes (fig. 6).

Il apparaît donc que le hapteur des Dactylogyridea possède trois types de pièces sclérifiées qui ont une origine différente :

- les crochetons larvaires qui arment le hapteur primitif ;

- les hamuli d'origine corporelle qui migrent secondairement dans le hapteur ;

- les barres transversales qui, bien que s'articulant avec les hamuli, ont une origine haptoriale. Lyons (1966) a d'ailleurs démontré que ces barres n'ont pas la même constitution chimique que les crochetons marginaux et les hamuli. Leurs scléroprotéines sont caractérisées par l'absence de cystéine, comme celles des pinces de Polyopisthocotylea ou des néoformations cuticulaires (pseudo-hapteurs, etc...).

A la suite de l'hypothèse que nous avons formulée sur la migration généralisée des onchoblastes, il apparaît des modalités communes dans la mise en place des hamuli 
chez tous les Dactylogyridea, ce qui traduit, d'après nous, leur unité phylétique. Les différences observées représentent des variations mineures (migration synchrone ou non des hamuli ventraux ou dorsaux, synthèse des scléroprotéines pendant ou après la migration des onchoblastes). Ces modalités se retrouvent aussi bien dans des familles qui ont un hapteur à 14 crochetons (Dactylogyridae, Diplectanidae, Ancyrocephalidae...) que chez les Tetraonchidae où il y a 16 crochetons larvaires.
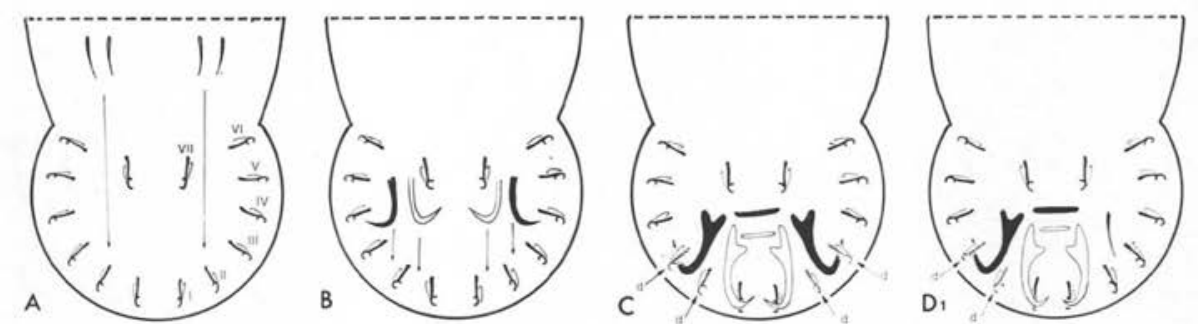

A.B.C. : Migration synchrone et nise en place des hamuli dorsaux (en noir) et

ventraux (en blane). (Vue ventrale).
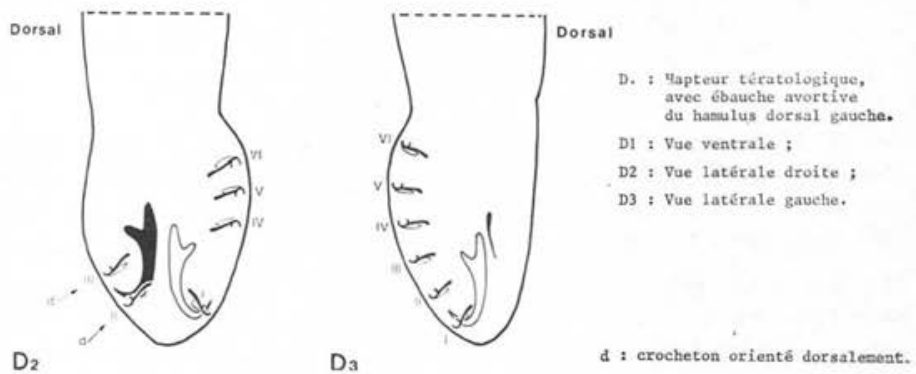

Fig. 6. Schéma du développement post-larvaire du hapteur chez Onchocleidus principalis.

Onchocleidus principalis : haptoral post-larval development.

b. Les transformations adaptatives du hapteur.

Le hapteur est à l'origine ventral, adapté à un attachement cutané ; c'est chez l'oncomiracidium qu'il est sous la forme la plus simple. La conquête de nouveaux microbiotopes par les Monogènes s'est traduite par des adaptations de l'organe de fixation. Nous allons voir ces transformations adaptatives chez les Dactylogyridea qui se sont diversifiés sur l'appareil branchial des Téléostéens.

L'attachement de l'adulte de Diplectanum aequans entre les lamelles branchiales de Dicentrarchus labrax a été étudié par Paling (1966). Kearn (1968 b) a décrit, chez cette espèce, "la métamorphose du parasite cutané en parasite branchial » et aussi, chez Neodactylogyrus crucifer. Cette transformation est la suivante: 
- Les ébauches des hamuli dorsaux, apparus dans le corps, sont, à l'origine, orientées ventralement.

- Après leur migration antéro-postérieure, elles effectuent dans le hapteur une rotation de $180^{\circ}$ sur leur axe, et leur pointe décrivant un demi-cercle latéral est finalement orientée dorsalement.

- Cette rotation entraîne, en position dorsale, les crochetons latéraux II et III, les autres crochetons restant orientés ventralement. Les barres transversales et les squamodisques (qui sont des néoformations cuticulaires) apparaissent par la suite.

La similitude des phénomènes observés chez Diplectanum aequans et Neodactylogyrus crucifer est suffisamment éloquente pour que Kearn y voit la preuve que, chez les Dactylogyridae, les hamuli ventraux sont représentés par une ébauche avortive (structure R).

Ces ébauches, qui se présentent sous la forme de fins sclérites associés aux crochetons I, ont été considérées par Mizelle et Price (1963) comme une paire de crochetons supplémentaires. Nous partageons l'opinion de Kearn, mais d'autres auteurs ne l'admettent pas (Gussev, 1967, 1976). Bien que Kearn $(1968 b$ ) n'ait pas pu suivre le développement complet de Tetraonchus monenteron, la seule comparaison du hapteur des post-larves et de celui de l'adulte (Kearn, 1966) laisse supposer que les choses se passent de la même manière chez ce Tetraonchidae.

Chez les Calceostomatidae, il n'y a pas de métamorphose du hapteur larvaire au sens de Kearn (Euzet et Ktari, 1973). En effet, l'attachement de l'adulte ne se fait pas entre deux lamelles branchiales; la face dorsale du hapteur n'intervient donc pas dans la fixation, et tous les crochetons larvaires restent orientés ventralement. Le rôle des hamuli dorsaux dans la fixation, s'il reste à préciser exactement, est peu important car ils ont disparu chez Calceostomella inerme qui s'attache à la branchie de la même manière que les espèces où ces hamuli persistent (Calceostoma ou Dicrumenia).

Le mode d'attachement est indépendant de la position systématique puisque d'après l'étude de la larve et du développement, Euzet et Ktari (1973) rapprochent les Calceostomatidae des Ancyrocephalidae.

Nos recherches ont porté essentiellement sur les Ancyrocephalidae qui représentent une "famille clé » des Dactylogyridae. Nous avons retrouvé certaines modalités de développement déjà observées par Kearn, mais aussi noté des différences. Dans tous les exemples que nous avons étudiés: Actinocleidus oculatus et A. recurvatus (Lambert, 1975), Ergenstrema mugilis (Lambert, 1977 a), Ancyrocephalus paradoxus (Lambert, $1977 b$ ), les hamuli dorsaux ont, dès leur origine corporelle, leur pointe orientée dorsalement et ils migrent ainsi dans le hapteur (4).

(4) Les préparations d'oncomiracidiums ou de post-larves imprégnés in to:o au nitrate d'Argent metient en évidence l'orientation de ces sclérites embryonnaires mieux que les o'servations sur le vivant. 
Kearn parle d'une migration de ces hamuli avec la pointe orientée ventralement puis d'une rotation, en direction dorsale. Selon nos observations, ce ne serait pas la rotation des hamuli dorsaux qui entraînerait le passage en position dorsale des crochetons II et III, mais leur arrivée au niveau de ces crochetons latéraux, sans que l'on puisse avancer une hypothèse pour expliquer ce passage dorsal des crochetons. Dans les deux cas, on aboutit au même résultat.

Ceci nous a amené à penser que la migration des hamuli, toujours orientés dorsalement, caractérisait les Ancyrocephalidae. Cependant, lorsque nous avons étudié les larves de Tetraonchus monenteron (Lambert, 1978 a) et les post-larves de Dactylogyrus extensus (Lambert, $1979 b$ ) pour l'étude de leurs structures argyrophiles, nous avons observé que chez ces Monogènes, l'ébauche des hamuli dorsaux était, dès leur origine corporelle, orientée dorsalement.

Chez Onchocleidus principalis, les ébauches des hamuli dorsaux et ventraux migrent ensemble de la région postérieure du corps vers le hapteur. Nous avons eu l'occasion d'observer, chez cet Ancyrocephalidae, une post-larve tératologique où les deux paires de hamuli sont déjà en place dans le hapteur mais il n'y a qu'un seul hamulus dorsal bien développé, celui du côté droit ; son symétrique, à l'état d'ébauche avortive, reste sous la forme d'un fin sclérite. Il semble que cet hamulus dorsal gauche a poursuivi sa migration depuis le corps sans que sa morphogénèse s'accomplisse (fig. 6). Du côté droit, les crochetons II et III sont orientés dorsalement alors que, du côté gauche, ils ont conservé l'orientation ventrale larvaire. Nous pouvons tirer de cette observation deux conclusions : tout d'abord, il semble que l'orientation dorsale des crochetons II et III est induite par le développement des hamuli dorsaux et non par un phénomène de rotation.

Nous pouvons supposer que le développement d'Eutrianchoratus Paperna, 1969 et Heteronchocleidus Bychowsky, 1957, dont les hapteurs adultes possèdent seulement trois hamuli, est de type Ancyrocephalidae (d'ailleurs, malgré un hapteur original, ces Monogènes ont une anatomie typique d'Ancyrocephalidae). On ne connaît pas le mode de fixation précis de ces Vers sur la branchie car l'assymétrie du hapteur perturbe la disposition des crochetons. Nous projetons d'étudier expérimentalement le développement post-larvaire de ces Monogènes pour comprendre les adaptations de cet attachement original.

Par rapport aux espèces que nous venons de citer, Ergenstrema mugilis présente une originalité certaine. Le mode de fixation de l'adulte, dont le microbiotope privilégié est le système des branchiospines, a été étudié et comparé à celui des Ligophorus Euzet et Suriano, 1977, qui sont aussi des parasites branchiaux de Mugilidae (Lambert et Sanfilippo, 1977). Le développement du hapteur d'Ergenstrema présente certaines convergences avec celui des Calceostomatidae : il n'y a pas de véritable métamorphose, car l'attachement n'intéresse pas la surface dorsale du hapteur, et il n'y a pas, au cours du développement post-larvaire, de changement dans l'orientation des crochetons qui restent tous ventraux. Les hamuli dorsaux existent, mais si, chez les Calceostomatidae, ils passent sur la face ventrale du hapteur, ils restent dorsaux chez Ergenstrema 
(fig. 7). Dans les deux cas, ils jouent un rôle effacé dans l'attachement qui se fait par une cupule ventrale où tous les crochetons ont gardé une orientation larvaire.

Chez Ergenstrema, au cours du développement, nous observons la migration des deux paires de hamuli. La filiation Ligophorus-Ergenstrema est évidente si nous comparons leur anatomie et nous avons là un exemple d'évolution adaptative du hapteur au microbiotope particulier que représentent les branchiospines (Lambert et Sanfilippo, 1977). Une particularité distingue Ergenstrema des autres Ancyrocephalidae: les
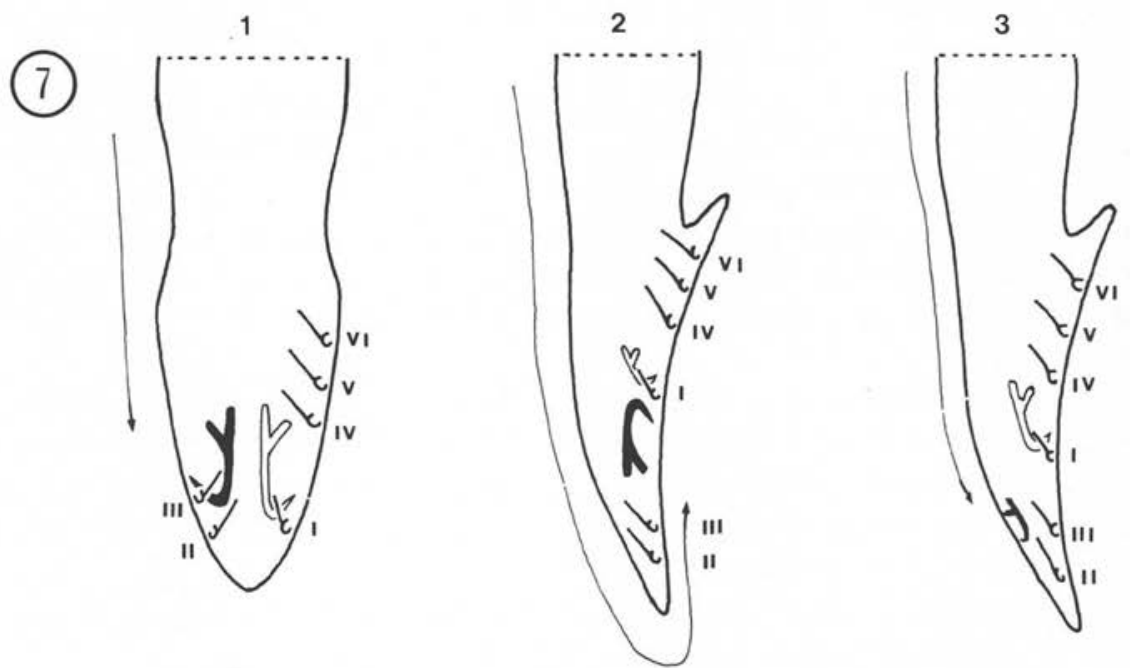

Fig. 7. Mise en place des hamuli dorsaux (en noir) chez trois Dactylogyridea. 1: Ancyrocephalidae branchiaux; 2: Calceostomatidae; 3: Ergenstrema mugilis.

Migration of the dorsal hamuli (in black) of three Dactylogyridea. 1: gill Ancyrocephalidae ; 2: Calceostomatidae ; 3 : Ergenstrema mugilis.

hamuli dorsaux, qui sont situés habituellement entre les crochetons II et III - ce qui, nous venons de le voir, est lié au passage de ces crochetons en position dorsale sont ici rejetés en position tout à fait postérieure, à la place des crochetons I. Ces derniers ont migré vers le centre du hapteur, en étroite association avec les hamuli ventraux. Les hamuli dorsaux qui ont, dès leur origine, une orientation dorsale, migrent, mais restent peu développés puisque leur taille est de l'ordre de celle des crochetons larvaires.

Il semble donc, contrairement à l'opinion de Kearn $(1968 b)$ et de Llewellyn (1968) que les hamuli dorsaux ne sont pas, à l'origine, des structures toujours orientées ventralement. Ceci est important sur le plan évolutif, car le phénomène pourrait être alors considéré comme une préadaptation à une fixation entre deux lamelles branchiales. Pourtant, les deux modalités ne seraient pas contradictoires si on admet que 
la rotation peut s'effectuer plus ou moins tôt lors de la migration des hamuli. Nous comprendrons mieux ces ontogénèses lorsqu'elles auront été étudiées chez des espèces dont le fonctionnement des hamuli est différent (Bifurcohaptor, Eutrianchoratus, Heteronchocleidus...).

A ce sujet, Euzetrema knoepffleri s'avère fondamentalement différent des Monogènes de Poissons. Chez ce parasite de l'Euprocte de Corse, qui possède deux paires de hamuli toutes deux orientées ventralement, on n'a pas pu déceler la migration antéro-postérieure de ces pièces (Combes, Jourdane et Knoepffler, 1974). Par contre, la paire de crochetons larvaires la plus postérieure (I) remonte pour occuper une place centrale dans le hapteur adulte au niveau des hamuli.

Ainsi, nous comprenons mieux comment, à partir d'un hapteur larvaire simple, se forment des organes de fixation très variés et diversifiés chez les adultes des Dactylogyridea. Cette diversité s'explique par:

1. L'acquisition des hamuli dorsaux qui peuvent influencer l'orientation de crochetons larvaires. Ils peuvent, suivant leur développement, jouer un rôle plus ou moins grand dans l'attachement par rapport à celui des hamuli ventraux (ex.: Bifurcohaptor, Nanotrema, etc...).

2. L'acquisition d'un système de pièces transversales entre les hamuli dorsaux et ventraux dont le nombre, le développement et le mode d'action sont très variables.

3. La modification et le développement de certains crochetons larvaires qui peuvent acquérir une fonction plus importante que les autres dans l'attachement (ex. : Dactylogyrus sphyrna ou D. vistulae).

4. La régression secondaire du système de fixation dorsal et retour à un attachement ventral de type cutané (Calceostomatidae, Ergenstrema).

5. La régression d'un seul hamulus d'où un attachement par trois hamuli (Eutrianchoratus, Heteronchocleidus...).

6. L'acquisition de néoformations cuticulaires (squamodisques des Diplectanum et Lamellodiscus, épines des Rhamnocercus).

A côté de cet ensemble homogène, il existe des familles comme les Gyrodactylidae, les Capsalidae, les Monocotylidae et les Acanthocotylidae qui sont fondamentalement des parasites cutanés, mais où rien n'indique une origine corporelle des hamuli (cette origine n'est pas à exclure si on admet une migration des onchoblastes et des recherches sont donc nécessaires sur l'origine et l'ontogénèse de ces pièces haptoriales en microscopie électronique). Cependant, ces parasites ont aussi conquis de nouveaux microbiotopes, car le hapteur ventral n'exclut pas une fixation superficielle sur l'épithélium branchial (Gyrodactylidae et Capsalidae). Trochopus pini, par exemple, enserre les lamelles branchiales et son hapteur entier joue à la manière d'une pince de Polyopisthocotylea (Kearn, 1971). Certains Monocotylidae sont endoparasites et on peut suivre la régression secondaire du hapteur chez des espèces comme Calicotyle kroyeri (cloaque des Raies), C. stossichi (glande rectale de Mustelus), Gymnocalicotyle inermis (oviducte) et Dictyocotyle coeliata (cavité générale). D’autres Monocotylidae 
(Horricauda), par l'acquisition de néoformations cuticulaires sur la face dorsale du hapteur, se sont adaptés à un microhabitat entre les lamelles branchiales (Kearn, 1978).

Quant aux Acanthocotylidae, le hapteur larvaire ne sert plus à la fixation de l'adulte qui a développé une néoformation cuticulaire.

\section{Conclusion.}

Les Monopisthocotylea assurent leur dispersion grâce à un oncomiracidium nageant qui possède un hapteur simple, ventral, adapté à la fixation sur une surface cutanée. On avait montré, et nous l'avons confirmé, que ces Monogènes envahissent leur hôte par la peau. Même si d'autres modalités, en particulier l'infestation passive par voie buccale, ne sont pas à exclure, l'existence d'une phase cutanée dans le cycle biologique des Monopisthocotylea branchiaux, quelle que soit son importance, est un argument en faveur de l'origine cutanée de ces Vers.

Chez les Polyopisthocotylea, où quelques espèces seulement ont été étudiées, les larves atteignent directement le microbiotope définitif : les branchies. Cela n'exclut pas cependant d'autres modalités. Chez les Polystomatidae par exemple, la larve infestante atteint les branchies du Têtard par le spiracle (Combes, 1968, 1972 b).

La niche écologique distingue également Monopisthocotylea et Polyopisthocotylea, les premiers mangent du mucus et des cellules cutanées, les seconds sont hématophages. Cette distinction se traduit, au niveau de l'intestin, par l'existence de deux types d'épithéliums (Halton et Jennings, 1965). Il semble donc exister une corrélation entre les modalités d'infestation et la nutrition de ces parasites. Ceux qui mangent des cellules épithéliales ont conservé une phase de leur cycle biologique passant par la peau de l'hôte; même s'ils sont secondairement branchiaux, ils ne sont jamais hématophages.

La distinction des deux sous-classes est aussi nette par le développement postlarvaire.

Chez les Polyopisthocotylea, Ktari (1971) a montré une unité dans ces modalités de développement que l'on a coutume de qualifier de «métamorphose». Certains crochetons larvaires disparaissent et il se forme de nouveaux organes (les pinces) qui fonctionnent de manière plus ou moins indépendante.

Chez les Monopisthocotylea, ces profondes transformations n'interviennent pas ; pourtant, il semble exister deux types de développement :

- le premier type : celui des Dactylogyridea, se retrouve chez les Dactylogyridae, les Ancyrocephalidae, les Diplectanidae, les Calceostomatidae et les Tetraonchidae;

- le deuxième type regroupe les Monocotylidae, les Capsalidae, les Acanthocotylidae et les Gyrodactylidae.

Pour les premiers, parasites secondaires des branchies, Kearn $(1968 b)$ a parlé de «métamorphose » du hapteur larvaire; il s'agit plutôt de remaniements induits par l'apparition de structures d'origine corporelle (les hamuli). Nous avons montré la 
nature adaptative de ces transformations qui dépendent du mode d'attachement et de la nature du substrat.

Dans le second type, il n'y a pas de remaniements importants car l'attachement reste de type cutané, ce qui n'exclut pas un parasitisme branchial.

Pour comparer rationnellement le développement du hapteur, il faudra établir les homologies de toutes les pièces qui le composent (et en particulier des hamuli) et voir si l'on peut généraliser l'hypothèse de la migration des onchoblastes.

La grande variabilité adaptative du hapteur, même larvaire, et notre ignorance des homologies entre les pièces qui le constituent, incitent à la prudence dans toute systématique élaborée presque exclusivement à partir de cet organe.

Si nous considérons le nombre de crochetons larvaires par exemple (dont on peut pourtant supposer qu'ils ont même origine), le hapteur à 16 crochetons est sans doute primitif, mais cela ne veut pas dire que ce caractère se retrouvera exclusivement conservé dans un seul phylum. Par exemple, Tetraonchus monenteron (à 16 crochetons) a un développement post-larvaire identique à celui des familles où il n'existe que 14 crochetons, et Polystoma (à 16 crochetons) subit une métamorphose du hapteur semblable à celle des Polyopisthocotylea.

Il s'avère nécessaire, pour établir des liens phylétiques entre les Monogènes, d'avoir de nouveaux arguments et donc d'ouvrir une nouvelle voie de recherches. Nos résultats sur l'étude des structures argyrophiles des oncomiracidiums répondent à cette nécessité.

\section{Bibliographie}

Baer I. G., Euzet L.: Classe des Monogènes. In: P.P. Grassé, Traité de Zoologic, 4, pp. 243-325. Masson édit., Paris, 1961.

Bauer O. N., Nikolskaya N.P. : Dactylogyrus solidus Akhmerov its biology development and significance in fish breeding (en Russe). Trudy Probl. Temat. Soveshch. Zool. Inst., 1954, 4, 99-109.

Bayssade-Dufour C. : L'appareil sensoriel des cercaires et la systématique des Trématodes digénétiques. Thèse Doct. Etat. Université Paris VI, 1978, 110.

Bovet J.: Contribution à la morphologie et à la biologie de Diplozoon paradoxum V. Nordmann, 1832. Bull. Soc. Neuchâtel, Sci. Nat., 1967, 90, 63-159.

Brooker B. E. : "The sense organs of trematode miracidia " in Behavioural asjects of parasite transmission. Zool. J. Lin. Soc., Supp. 1, 1972, 51, 171-180.

Bychowsky B.E.: Bermerkungen über dic monogenetischen Trematoden der Gattung Dactylogyrus Dies. der in den Teichen des Schutzsparken des Peterhofer Biologischen Institutes wohnender Karauschen (en Russe). Trudy Leningr. Obshch. Estest. Zool., 1933, 62, 269-296.

Bychowsky B. E. : Monogenetic Trematodes, their systematics and phylogeny (en Russe). Akad. Nauk. C.C.C.P., 1957, 1-509. English Transl. AIBS Washington, W. Hargis (1961).

Bychowsky B. E., Nagibina L. F. : On «intermediate host " in Monogeneans (Monogenoidea) (en Russe). Parazitologiia, 1967, 1, 117-124.

Chatton E., Lwoff A.: Imprégnation, par diffusion argentique, de l'infraciliature des ciliés marins et d'eau douce après fixation cytologique et sans dessication. C.R. Soc. Biol., 1930, 104, 834-836.

Combes C.: Biologie, écologie des cycles et biogéographie des Digènes et Monogènes d'Amphibiens dans l'est des Pyrénées. Mem. Mus. Nat. Hist. Nat. Sér. A., 1968, 51, 1-195.

Combes C.: Ecologie des Polystomatidae (Monogenea). Facteurs influençant le volume et le rythme de la ponte Inter. J. Parasitol., 1972 a, 2, 233-238. 
Combes C.: Influence of the behaviour of amphibians of helminth life. In: «Behavioural aspects of Parasites transmission ». C.A. Wright., Edit. Zool. J. Lin. Soc. Suppt 1, 1972 b, 51, 151-170.

Combes C., Bayssade-Dufour C., Cassone J.: Sur l'imprégnation et le montage des cercaires pour l'étude chétotaxique. Ann. Parasitol. Hum. Comp., 1976, 51, 399-400.

Combes C., Jourdane J., Knoepffler L.P.: Le cycle biologique de Euzetrema knoepffleri Combes, 1965 (Monogenea, Monopisthocotylea), parasite de l'Euprocte de Corse. Bull. Soc. Zool. Fr., 1974, 99, $219-236$.

Combes C., Lambert A. : Evolution de l'appareil sensoriel au cours de la vie larvaire chez les Monogènes Polyopisthocotylea. C.R. Acad. Sci., 1972, 274, 402-404.

Combes C., Lambert A.: La chétotaxie des larves nageantes de Polystomes européens (Monogenea). Ann. Parasitol. Hum. Comp., 1975, 50, 25-37.

Combes C., Lambert A., Bourgat R., Salami-Cadoux M.-L.: L'oncomiracidium de Eupolystoma alluaudi (De Beauchamp, 1913) Monogène parasite de Bufo regularis Reuss en Afrique. Bull. Nat. Hist. Nat., Zool., 1978, 353, 211-216.

Croghan P.C.: The mecanism of osmotic regulation in Artemia salina (L.): the physiology of the branchiae. J. Exp. Biol., 1958, 35, 234-249.

Dixon K.E., Mercer E.H.: The fine structure of the nervous system of the cercaria of the liver fluke Fasciola hepatica. J. Parasitol., 1965, 51, 967-976.

Erasmus D. A.: The host-parasite interface of Cyathocotyle bushiensis Khan 1962 (Trematoda Strigeoidea) II. Electron miscroscope studies of the tegument. J. Parasitol., 1967, 53, 703-714.

Euzet L.: Larves gyrodactyloïdes nageantes de quelques Trématodes Monogénétiques de Poissons marins. Bull. Soc. Neuchâtel, Sci. Nat., 1955, 78, 71-79.

Euzet L.: Recherches sur les Monogenoidea parasites de Poissons marins. Ann. Parasitol. Hum. Comp.. $1957,32,469-481$.

Euzet L. : Sur le développement post-larvaire des Microcotylidae (Monogenoidea Polyopisthocotylea). Bull. Soc. Neuchâtel, Sci. Nat., 1958, 81, 79-84.

Euzet L., Ktari M. H. : Le développement larvaire et la position systématique des Calcéostomatidae (Monogenea). C.R. Acad. Sci. Paris, 1970, 271, 2332-2335.

Euzet L., Ktari M.H.: Les Calceostomatidae (Monogenea) parasites de Téléostéens du Golfe de Tunis. Développement larvaire et position systématique. Bull. Inst. Oceanogr. Pêche, Salammbô, 1973, 2, 605-622.

Euzet L., Lambert A. : Compléments à l'étude de la larve de Diplozoon paradoxum Von Nordmann, 1832 (Monogenea). Ann. Parasitol. Hum. Comp., 1971, 46, 675-684.

Euzet L., Lambert A.: Chétotaxie comparée des larves de Diplozoon paradoxum Von Nordmann 1832 et de Diplozoon gracile Reichenbach-Klinke, 1961 (Monogenea). Bull. Soc. Zool. Fr., 1974. $99,307-314$

Ginetsinkaya T. A., Dobrovolsky A. A.: Une nouvelle méthode pour mettre en évidence les sensilles chez les larves de Trématodes et le rôle de ces structures dans la taxonomie (en Russe). Dokl. Akad. Nauk. S.S.S.R., 1963, 151, 460-463.

Gröben G. : Beobachtungen über die Entwicklung verschiedener Arten von Fischschnarotzern aus der Gattung Dactylogyrus. Z. Parasitenk., 1940, 11, 611-636.

Gussev A. V.: The morphological criterian and characters of freshwater monogenetic trematodes in modern taxonomy (en Russe). Parassitolozia, 1967, 1, 55-67.

Gussev A.V.: Freshwater indian monogenoidea; Principles of systematics, analysis of the world faunas and their evolution. Ind. J. Helminth., 1976 (1973-1974), 25-26, 1-241.

Gussev A.V., Kulemina I. V.: Analysis of variability of characters, behaviour and development's cycle of Monogeneans from the age of hosts (en Russe). Parasitologiya, 1971, 5, 320-329.

Halton D. W., Jennings J. B.: Observations on the nutrition of Monogenetic trematodes. Biol. Bull., $1965,129,257-272$.

Hargis W. J. : Monogenetic trematodes of Gulf of Mexico Fishes. Part. I. The superfamily Gyrodactyloidea. Biol. Bull., 1955, 108, 125-137.

Hargis W. J.: Monogenetic trematodes of Gulf of Mexico Fishes. Part. XIII. The family Gastrocotylidae, Price 1943. Trans. Amer. Microsc. Soc., 1957, 76, 1-12. 
Iziumova N. A. : Material on the biology of Dactylogyrus vastator Nybelin (en Russe). Parasitol. Sb Zool. Inst. Akad. Nauk. S.S.S.R., 1956, 16, 229-243.

Kearn G.C.: The egg, oncomiracidium and larval development of Entobdella soleae a monogenean skin parasite of the common Sole. Parasitology, $1963 a, 53,435-447$.

Kearn G.C.: The life cycle of the monogenean Entobdella soleae a skin parasite of the common Sole. Parasitology, 1963 b, 53, 253-263.

Kearn G.C.: The adhesive mechanism of the monogenean parasite Tetraonchus monenteron from the gills of the pike (Esox lucius). Parasitology, 1966, 56, 505-510.

Kearn G.C.: The life cycles and larval development of some acanthocotylids (Monogenea) from Plymouth rays. Parasitology, 1967, 57, 157-167.

Kearn G.C.: The larval development of Merizocotyle sp. a monocotylid monogean from the nasal fossae of Raia undulata. Parasitology, 1968 a, 58, 921-928.

Kearn G.C.: The development of the adhesive organs of some diplectanid, tetraonchid and dactylogyrid gill parasites (Monogenea). Parasitology, $1968 b$, 58, 149-163.

Kearn G.C.: The attachment site, invasion route and larval development of Trochopus pini, a monogenean from the gills of Trigla hirundo. Parasitology, 1971, 63, 513-525.

Kearn G.C.: Early development and microhabitat of the monogenean Horricauda rhinobatidis, with observations on the related Troglocephalus rhinobatidis from Rhinobatos batillum from Queensland, Australia. Int. J. Parasitol., 1978 a, 8, 305-311.

Ktari M.H.: Recherches sur la preproduction et le développement de quelques Monogènes (Polyopisthocotylea) parasites de Poissons marins. Thèse Doctorat Etat, $\mathrm{n}^{\circ}$ C.N.R.S. AO 6332, U.S.T.L. Montpellier, 1971.

Ktari M.H.: The occurence of two congeneric species of Monogenea on the gills of Echeneis naucrates L. (Pisces-Teleostei) collected in Tunisia. Second European Multicolloquy of Parasitology. Trogir 1-6 sept. 1975. Summaries: 93-94, 1975.

Ktari M. H. : Le parasitisme d'Echeneis naucrates L. (Poisson Téléostéen) par deux Monogènes (Monopisthocotylea) du genre Dionchus: D. agassizi Goto 1899 et D. remorae MacCallum 1916. Excerta Parasit. en memoria del Dr. E. Caballero y Caballero, Inst. Biol., 1977, 61-67.

Lajman E. M. : The influence of the growth of the Carp on its infectivity with parasites (en Russe). Gel'minth. Sb. Deiatel'nost. Skrjabin, 1946, 171-177.

Lambert A.: Développement post-larvaire d'Actinocleidus sp. Mueller 1937 (Monogenea, Monopisthocotylea, Ancyrocephalidae). Hypothèse de la migration des onchoblastes chez les Dactylogyroidea. C.R. Acad. Sci., 1975, 281, 1329-1332.

Lambert A. : Mise en évidence et importance de la chétotaxie larvaire chez les Monogènes Monopisthocotylea. C.R. Acad. Sci., 1976, 282, 1109-1112.

Lambert A.: Développement larvaire et post-larvaire d'Ergenstrema mugilis Paperna, 1964 (Monogenea, Ancyrocephalidae) parasite de Liza ramada (Risso, 1826) (Téléostéen, Mugilidae). Z. Parasitenk., 1977 a, 52, 229-240.

Lambert A. : L'oncomiracidium d'Ancyrocephalus paradoxus Creplin, 1839 (Monogenea, Monopisthocotylea) parasite de Sander lucioperca (Téléostéen, Percidae). Ann. Parasitol. Hum. Comp., $1977 b, 52,493-505$.

Lambert A.: Recherches sur les affinités phylétiques des Polystomatidae. C.R. Acad. Sci., 1977 c, $285,1243-1246$.

Lambert A.: Précisions sur l'oncomiracidium de Tetraonchus monenteron (Wagener, 1857) Diesing 1858 (Monogenea, Tetraonchidae) parasite d'Esox lucius L. (Téléostéen). Ann. Parasitol. Hum. Comp., 1978 a, 53, 117-121.

Lambert A. : Chétotaxie larvaire et phylogénie des Monogènes. Fourth. Inter. Cong. of Parasitology, Warszawa 19-26 août 1978 , Sec. $B_{2}: 25-26$.

Lambert A.: Recherches sur l'oncomiracidium des Capsalidae (Monogena). Ann. Parasitol. Hum. Comp., 1978 c, 53, 351-357.

Lambert A.: Recherches sur les stades larvaires des Monogènes de Poissons. Ann. Parasitol. Hum. Comp., $1978 d, 53,551-559$.

Lambert A.: Recherches sur les affinités phylétiques des Gyrodactylidea (Monogenea). C.R. Acad. Sci., $1979 a, 288,231-233$. 
Lambert A.: Evolution post-larvaire de l'appareil sensoriel chez les Dactylogyridea (Monogenea). Z. Parasitenk., 1979 b, 58, 259-263.

Lambert A., Bourgat R. : L'oncomiracidium de Metapolystoma brygoonis (Euzet et Combes, 1964) parasite de l'Amphibien malgache Ptychadena mascareniensis (Dumeril et Bribon). Ann. Parasitol. Hum. Comp., 1978, 53, 547-549.

Lambert A., Combes C., Ktari M.H.: Morphologie de l'oncomiracidium de Polystomoïdes Ward, 1917 (Monogenea) et situation du genre parmi les Polystomatidac. Z. Parasitenk., 1978, 56, 175-181.

Lambert A., Maillard C. : Parasitisme branchial simultané par deux espèces de Diplectanum Diesing 1858 (Monogenea, Monopisthocotylea) chez Dicentrarchus labrax (L.) (Téléostéen). C.R. Acad. Sci., 1974, 279, 1345-1347.

Lambert A., Maillard C. : Répartition branchiale de deux Monogènes : Diplectanum aequans (Wagener, 1857) Diesing 1858 et Diplectanum laubieri Lambert et Maillard, 1974 (Monogenea, Monopisthocotylea) parasites simultanés de Dicentrarchus labrax (Téléostéen). Ann. Parasitol. Hum. Comp., 1975, 50, 691-699.

Lambert A., Maillard C.: L'oncomiracidium d'Erpocotyle catenulata (Guberlet, 1933) (Monogène Hexabothriidae) parasite de Mustelus mustelus L. (Selachii). Ann. Parasitol. Hum. Comp., 1979, 54, 113-115.

Lambert A., Sanfilippo D.: Position systématique et biologie d'Ergenstrema mugilis Paperna, 1964 (Monogenea, Monopisthocotylea) parasite de Liza (Liza) ramada (Risso, 1826) (Téléostéen, Mugilidae). Bull. Mus. Nat. Hist. Nat. Zool, 1977, 329, 823-831.

Lie K. J.: Studies on Echinostomatidae (Trematoda) in Malaya. XIII. Integumentary papillae on six species of Echinostome cercariae. J. Parasitol., 1966, 52, 1041-1048.

Llewellyn J.: The larvae of some Monogenetic Trematode Parasites of Plymouth fishes. J. mar. biol. Ass. U.K., 1957 a, 36, 243-259.

Llewellyn J.: Host specificity in Monogenetic Trematodes. In : First Symposium on host specificity among Parasites of Vertebrates. U.I.S.B. et Université de Neuchâtel Pub., 1957 b, 199-211.

Llewellyn J. : Larvae and larval development of Monogeneans. Adv. Parasitol., 1963, 1, 287-326.

Llewellyn J. : Larvae and larval development of Monogeneans. Adv. Parasitol., 1968, 6, 373-383.

Llewellyn J.: Behaviour of monogeneans. In : Behavioural aspects of parasitic transmission. Zool. $J$. Lin. Soc., Suppt, 1, 1972, 51, 19-30.

Lynch J. E.: The miracidium of Heronimus chelydrae MacCallum. Quart. J. Micr. Sci. London, 1933, 76, $13-33$.

Lyons K. M. : The chemical nature and evolutionary significance of Monogenean attachment sclerites. Parasitology, 1966, 56, 63-100.

Lyons K. M. : Sense organs of Monogenean skin parasites ending in a typical cilium. Parasitology, $1969 a, 59,611-623$.

Lyons K. M. : Compound sensilla in Monogenean skin parasites. Parasitology, 1969 b, 59, 625-636.

Lyons K. M.: Sense organs in Monogeneans. In: Behavioural aspects of parasites transmission. Zool. J. Linn. Soc. Suppt 1, 1972 a, 51, 181-199.

Lyons K. M.: Scanning and transmission electron microscope studies on the sensory sucker papillae of the fish parasite Entobdella soleae (Monogenea). Z. Zellforsch., 1973 a, 137, 471-480.

Matricon-Gondran M.: Etude ultrastructurale des récepteurs sensoriels tégumentaires de quelques Trématodes digénétiques larvaires. Z. Parasitenk., 1971, 36, 318-333.

Mizelle J.D., Price C. E. : Additional haptoral hooks in the genus Dactylogyrus. J. Parasitol., 1963, 49, 1028-1029.

Morris G.P., Threadgold L. T.: A presumed sensory structure associated with the tegument of Schistosoma mansoni. J. Parasitol., 1967, 53, 537-539.

Oliver G. : Nouvelles observations sur la biologie et l'écologie de quelques Diplectanidae (Monogenea, Monopisthocotylea) (en Russe). In: Volume à la mémoire de B. E. Bychowsky, Akad. Nauk. C.C.C.P., 1976, 34, 104-109.

Paling J.E.: The attachment of the monogenean Diplectanum aequans (Wagener) Diesing to the gills of Morone labrax L. Parasitology, 1966, 56, 493-503. 
Paling J.E.: The manner of infection of Trout gills by the monogenean parasite Discocotyle sagittata. J. Zool. London, 1969, 159, 293-309.

Paperna I.: Some observations on the biology and ecology of Dactylogyrus vastator in Israël. Bamidgeh Bull. Fish. Cult., 1963, 15, 8-28.

Price E. W. : North american monogenetic trematodes. I. The superfamily Gyrodactyloidea. J. Wash. Acad. Sci., 1937, 27, 114-130.

Price E. W.: North American monogenetic trematodes. XI. The family Heteraxinidae. J. Parasitol., 1962, 48, 402-418.

Prost M.: Investigations on the development and pathogenicity of Dactylogyrus anchoratus (Duj. 1845) and D. extensus Mueller et Van Cleave 1932 for breeding carps. Acta Parasitol. Pol., 1963, $11,17-47$.

Richard J.: La chétotaxie des cercaires. Valeur systématique et phylétique. Mem. Mus. Nat. Hist. Nat. Sér. A, Zool., 1971, 67, 1-179.

Rohde K. : Sense receptors of Multicotyle purvisi Dawes (Trematoda, Aspidobothria). Nature, Lond., 1966, 211, 820-822.

Rohde K.: Lichtmikroskopische Untersuchungen an den Sinnesrezeptoren der Trematoden. Z. Parasitenk., 1968, 30, 252-277.

Rohde K.: The Aspidogastrea, especially Multicotyle purvisi Dawes, 1941. Adv. Parasitol., 1972, 10, 77-151.

Sproston N. G. : A synopsis of the Monogenetic Trematodes. Trans. Zool. Soc. London, 1946, 25, 185-600.

Wagner A.: Papillae on three species of Schistosome cercariae. J. Parasit., 1961, 47, 614-618.

Wilson R. A.: Fine structure of the nervous system and specialized nerve endings in the miracidium of Fasciola hepatica. Parasitology, 1970, 60, 399-410.

Wunder W.: Die Dactylogyruskrankheit der Karpfenbrut, ihre Ursache und ihre Bekämpfung. Z. Fish., 1929, 27, 511-545. 